TRANSACTIONS OF THE

AMERICAN MATHEMATICAL SOCIETY

Volume 352 Number 4, Pages 1825-1858

S 0002-9947(99)02352-1

Article electronically published on October 29, 1999

\title{
VARYING BIFURCATION DIAGRAMS OF POSITIVE SOLUTIONS FOR A CLASS OF INDEFINITE SUPERLINEAR BOUNDARY VALUE PROBLEMS
}

\author{
JULIÁN LÓPEZ-GÓMEZ
}

\begin{abstract}
In this work we analyze the existence, multiplicity and stability of positive solutions for a class of indefinite superlinear elliptic boundary value problems. The main contribution of this paper consists in the change of mind inherent to the fact of adding the superlinear amplitude $\varepsilon$ as an unfolding parameter. This change of mind allows us to unify many previous results obtained separately in the literature, it helps us to realize the global structure of the set of positive steady states, and provides us with a great variety of new general results. Our techniques can be applied to much more general equations and systems.
\end{abstract}

\section{IntRoduction}

In this work we analyze the existence, multiplicity and stability of positive steady states of the following reaction diffusion problem

$$
\begin{gathered}
\partial_{t} u+\mathcal{L} u=\lambda u-a_{\varepsilon}(x) u^{2} \quad \text { in } \Omega \times(0, \infty), \\
u=0 \quad \text { on } \partial \Omega \times(0, \infty), \\
u(\cdot, 0)=u_{0} \geq 0 \quad \text { in } \bar{\Omega},
\end{gathered}
$$

where $\Omega$ is a bounded domain of $\mathbb{R}^{N}, N \geq 1$, with boundary $\partial \Omega$ of class $C^{2+\nu}$ for some $\nu \in(0,1), \mathcal{L}$ is a second order uniformly elliptic operator of the form

$$
\mathcal{L}:=-\sum_{i, j=1}^{N} D_{i}\left(c_{i j}(x) D_{j}\right)+c_{0}(x),
$$

with $c_{i j} \in C^{1+\nu}(\bar{\Omega}), c_{i j}=c_{j i}, i, j=1, \ldots, N, c_{0} \in C^{\nu}(\bar{\Omega}), \lambda \in \mathbb{R}$ is a real parameter, and $a_{\varepsilon} \in C^{\nu}(\bar{\Omega})$ is an indefinite weight function of the form

$$
a_{\varepsilon}=a^{+}-\varepsilon a^{-},
$$

where $\varepsilon \geq 0, a^{+}, a^{-} \in C^{\nu}(\bar{\Omega})$, and

$$
\Omega_{+}:=\left\{x \in \Omega: a^{+}(x)>0\right\}, \quad \Omega_{-}:=\left\{x \in \Omega: a^{-}(x)>0\right\}
$$

Received by the editors August 26, 1996 and, in revised form, October 14, 1997.

2000 Mathematics Subject Classification. Primary 35B60, 35J25, 35K20.

Key words and phrases. Indefinite superlinear problems, superlinear amplitude as unfolding parameter, multiplicity results without a priori bounds.

This work was supported by the EC Network REACTION DIFFUSION EQUATIONS under grant ECBCHRX CT 93-0409 and the Spanish DGYCIT PB93-0465 and DGES PB96-0621. 
are disjoint subdomains of $\Omega$ whose boundaries have the same regularity as $\partial \Omega$ and $\bar{\Omega}_{+} \subset \Omega, \bar{\Omega}_{-} \subset \Omega$. In particular, $a_{\varepsilon}=0$ in

$$
\Omega_{0}:=\Omega \backslash\left(\bar{\Omega}_{+} \cup \bar{\Omega}_{-}\right)
$$

We shall refer to $\varepsilon$ as a parameter measuring the amplitude of the superlinear part of the nonlinearity.

Problem (1.1) is well-posed in the Banach space $U:=C_{0}^{2+\nu}(\bar{\Omega})$, the closed subspace of $C^{2+\nu}(\bar{\Omega})$ constituted by the functions $u$ vanishing on $\partial \Omega$. By the parabolic maximum principle, if $u_{0}>0$, then the solution $u\left(t, x ; u_{0}\right)$ of $(1.1)$ lies in the interior of the positive cone of $C_{0}^{1}(\bar{\Omega})$ for all $t>0$ where it exists.

The positive steady states of (1.1) are the positive solutions of

$$
\mathcal{L} u=\lambda u-a_{\varepsilon}(x) u^{2}, \quad u \in U
$$

When $\varepsilon=0$, then $a=a^{+}$and in this special case the structure of the set of positive solutions of (1.3) is well known. Namely, an increasing analytic curve bifurcating from $u=0$ at $\lambda=\sigma_{1}^{\Omega}[\mathcal{L}]$ and growing to infinity as $\lambda \uparrow \sigma_{1}^{\Omega \backslash \bar{\Omega}_{+}}[\mathcal{L}]$, 7], 22], [11, 14]. Hereafter, $\sigma_{1}^{D}[\mathcal{L}]$ stands for the principal eigenvalue of $\mathcal{L}$ in $D \subset \Omega$ under homogeneous Dirichlet boundary conditions. The techniques introduced in [14 also provide us with the existence and some uniqueness results for a very general class of sublinear elliptic boundary value problems 20 .

When $\varepsilon>0$ then a sort of competition between the sub and the superlinear parts of the nonlinearity arises, making the analysis of the existence and multiplicity of positive steady states far from easy, even looking as simple as (1.3) does. In the last few years three types of techniques have been used, and sometimes developed, to treat this problem.

Ouyang [23] took $\mathcal{L}=-\Delta$ and using some classical techniques of bifurcation theory showed that if the spatial dimension is not too large and the bifurcation to positive solutions from $u=0$ is supercritical, then there exists $\lambda_{0} \in\left(\sigma_{1}^{\Omega}[\mathcal{L}], \sigma_{1}^{\Omega \backslash \bar{\Omega}_{+}}[\mathcal{L}]\right)$ such that (1.3) possesses at least two positive solutions for each $\lambda \in\left(\sigma_{1}^{\Omega}[\mathcal{L}], \lambda_{0}\right)$ and it has no solution for $\lambda>\lambda_{0}$.

Beresticky, Capuzzo-Dolcetta and Nirenberg in [3], 4] and [5], using variational methods and topological degree, found some existence results. Some of them are of a local nature, but others are global and those will be used in Section 6 to get some new multiplicity results. More precisely, we shall make use of the a priori bounds of [5]. We refer to Section 6 for a detailed comparison of our results versus the results of [5]. In Section 2 will be given a complete answer to all the questions that arise in the final remarks of [4]. I would advise having copies of these papers when reading this one.

Alama and Del Pino [1, using Morse theory and min-max methods, found some multiplicity results, but their solutions possibly change sign and therefore their results are useless for our purposes herein.

The main contribution of this paper consists in the change of mind inherent to the fact of adding the superlinear amplitude $\varepsilon$ as an unfolding parameter. This change of mind allows us to unify many previous results obtained separately in the literature and helps us to realize the global structure of the set of positive solutions 
of (1.3). For example, by setting

$$
\varepsilon^{*}:=\frac{\int_{\Omega_{+}} a^{+} \varphi_{1}^{3}}{\int_{\Omega_{-}} a^{-} \varphi_{1}^{3}}
$$

where $\varphi_{1} \gg 0$ stands for the principal eigenfunction (positive) associated with $\sigma_{1}^{\Omega}[\mathcal{L}]$, Theorems 2 and 3 of Beresticky, Capuzzo-Dolceta and Nirenberg [4] are better understood in the following terms: The bifurcation to positive solutions from $u=0$ is supercritical if $\varepsilon<\varepsilon^{*}$, and subcritical if $\varepsilon \geq \varepsilon^{*}$. This provides us with a new and striking proof of Theorem 3 of 4 .

Once the new parameter $\varepsilon$ has been introduced, a very natural question arises. How does the global continuum of positive solutions of (1.3) vary when $\varepsilon$ changes from 0 to infinity? Giving a complete answer to this question should provide us with a unified view over all previous results and so it should help us to find out new and deeper results. This is what we are going to do in this paper. Our strategy will be the following. First, to analyze (1.3) when $\varepsilon$ is small, then to perform a global continuation using $\varepsilon$ as the main parameter.

This paper is organized as follows. In Section 2 we introduce some notations, recall the characterization of the strong maximum principle by means of strict positive supersolutions, analyze the behaviour of (1.3) when $\varepsilon=0$ and then give the first general results about the existence of positive solutions of (1.3). A new and shorter proof of the main theorems of [7], [22] and [14] will be given, analyzing, in addition, how the solution grows to infinity as $\lambda \uparrow \sigma_{1}^{\Omega \backslash \bar{\Omega}_{+}}[\mathcal{L}]$, [11]. We need this growth to show that there exists $\lambda^{*}(\varepsilon)<\sigma_{1}^{\Omega \backslash \bar{\Omega}_{+}}[\mathcal{L}]$ such that (1.3) does not admit a positive solution if $\lambda>\lambda^{*}(\varepsilon)$ and, in addition, to get explicit estimates for $\lambda^{*}(\varepsilon)$. These estimates show how $\lambda^{*}(\varepsilon)$ decreases as $\varepsilon$ increases. Moreover, it will be shown that for any

$$
\delta \in\left(0, \sigma_{1}^{\Omega \backslash \bar{\Omega}_{+}}[\mathcal{L}]-\sigma_{1}^{\Omega}[\mathcal{L}]\right)
$$

there exists $\varepsilon(\delta)>0$ such that (1.3) possesses a positive solution for each

$$
\lambda \in\left(\sigma_{1}^{\Omega}[\mathcal{L}], \sigma_{1}^{\Omega \backslash \bar{\Omega}_{+}}[\mathcal{L}]-\delta\right)
$$

and $\varepsilon \leq \varepsilon(\delta)$. However, to be rather natural, all these results are new. Also, we should point out that none of them depends qualitatively on the spatial dimension, as it happens with most of the existence results in the references.

In Section 3 we use singularity theory techniques to analyze the nature of the local bifurcation to positive solutions from $u=0$. If $\varepsilon>\varepsilon^{*}$, then the bifurcation is transcritical with the half curve of positive solutions emanating subcritically, while if $\varepsilon=\varepsilon^{*}$, then the bifurcation is a subcritical quadratic pitchfork, and if $\varepsilon<\varepsilon^{*}$, then it is transcritical with the positive solutions emanating supercritically. Since the pitchfork obtained when $\varepsilon=\varepsilon^{*}$ is quadratic, it follows from the theory of Golubitsky and Schaeffer [15] that for $\varepsilon<\varepsilon^{*}$ close enough there exists $\lambda[\varepsilon]>\sigma_{1}^{\Omega}[\mathcal{L}]$ such that in a neighborhood of $(u, \lambda)=\left(0, \sigma_{1}^{\Omega}[\mathcal{L}]\right)$ the problem $(1.3)$ does not admit a positive solution if $\lambda>\lambda[\varepsilon]$, while it has one if $\lambda=\lambda[\varepsilon]$ and it has two for each $\lambda \in\left(\sigma_{1}^{\Omega}[\mathcal{L}], \lambda[\varepsilon]\right)$. In the latest case the smaller solution is linearly asymptotically stable while the other is unstable with one-dimensional unstable manifold (cf. Figure 3 in Section 3). A fundamental observation is that this result is independent of the spatial dimension. When the spatial dimension is sufficiently low, then a priori bounds are available in the region where $u=0$ is unstable and 
therefore for each $\varepsilon<\varepsilon^{*}$ there exists $\lambda_{1}>\sigma_{1}^{\Omega}[\mathcal{L}]$ such that (1.3) possesses at least two solutions if $\lambda \in\left(\sigma_{1}^{\Omega}[\mathcal{L}], \lambda_{1}\right)$, being the minimal one weakly stable in the sense of Amann [2].

Singularity theory has proven so useful because it suggests the local behaviour persists for values of the parameters far away from their singular values. Model (1.3) provides us with an excellent example of this. For example, it does not admit a positive solution if $\varepsilon \geq \varepsilon^{*}$ and $\lambda \geq \sigma_{1}^{\Omega}[\mathcal{L}]$ (cf. Theorem 3.6 in Section 3). This result is a generalization of Theorem 2 of [4]. As in 4] it follows from a Picone identity [4], 24]. Moreover, the local bifurcation diagram obtained for $\varepsilon<\varepsilon^{*}$ sufficiently close still persists as $\varepsilon$ decreases up to reach a critical value $\varepsilon_{*} \in\left[0, \varepsilon^{*}\right)$, where the $u$ components of the turning points grow to infinity while their $\lambda$ components increase converging to a value $\lambda_{1} \leq \sigma_{1}^{\Omega \backslash \bar{\Omega}_{+}}[\mathcal{L}]$. At $\varepsilon=\varepsilon_{*}$ the global continuum of positive solutions of (1.3) bifurcating from $u=0$ is formed by an increasing analytical curve filled in by linearly asymptotically stable solutions blowing up as $\lambda \uparrow \lambda_{1}$. This global theorem is one of the main results in this paper. Its proof is included in Section 5, and it is based upon the direct method for computing higher order folds proposed by Keller and Yang in [16] and the theory previously developed in Section 4 , where we characterize whether or not a singular point is a single fold. Since this is the first global analytical result of this nature available in the literature in the context of problem (1.3), at a first glance, its reading might be a bit confusing for nonexperts in the numerical analysis of bifurcation problems.

In general, we do not know whether $\varepsilon_{*}=0$ or $\varepsilon_{*}>0$ occurs. If a priori bounds for the positive solutions of (1.3) are available for the range of $\lambda$ 's where the trivial solution is unstable, then $\varepsilon_{*}=0$. If not, $\varepsilon_{*}>0$ might occur. Therefore, the spatial dimension seems to play an important role to ascertain whether $\varepsilon_{*}>0$ or $\varepsilon_{*}=0$ occurs. In fact, thanks to the results of Beresticky, Capuzzo-Dolcetta and Nirenberg [5] and Ouyang [23, if the spatial dimension is sufficiently low, then $\varepsilon_{*}=0$. In other words, the local subcritical turning point emanating from the bifurcation point at $\varepsilon=\varepsilon^{*}$ persists for all values $\varepsilon \in\left(0, \varepsilon^{*}\right)$. Moreover, if we denote the turning point by $(u[\varepsilon], \lambda[\varepsilon])$, then

$$
\lim _{\varepsilon \downarrow 0} u[\varepsilon]=\infty
$$

uniformly on compact subsets of $\Omega \backslash \bar{\Omega}_{+}$, and

$$
\lim _{\varepsilon \downarrow 0} \lambda[\varepsilon]=\sigma_{1}^{\Omega \backslash \bar{\Omega}_{+}}[\mathcal{L}]
$$

(cf. Section 5 for details). Therefore, in the presence of a priori bounds the branch of minimal solutions of $(1.3)$, those asymptotically stable connecting $\left(0, \sigma_{1}^{\Omega}[\mathcal{L}]\right)$ with the subcritical turning point $(u[\varepsilon], \lambda[\varepsilon])$, converges as $\varepsilon \downarrow 0$ to the global bifurcation diagram of (1.3) with $\varepsilon=0$. This seems to be the first global analytical result of this nature available in the literature.

In Section 6 we shall complete the comparison of our results with those found in [5] and [23], giving, in addition, some further global results.

Most of our results are true for general differential operators $\mathcal{L}$, not necessarily selfadjoint, general boundary conditions (cf. [14]), and much more general nonlinearities. We have chosen $\mathcal{L}$ selfadjoint for the validity of the Picone identity. We have chosen $u^{2}$, instead of $u^{p}, p>1$, because in this special situation the singularity theory needed to treat (1.3) is contained in [15], while in the general case we 
should elaborate more on this point. Our techniques also apply for more general nonlinearities like $a^{+} u^{p}-\varepsilon a^{-} u^{q}$ with $p \neq q$.

\section{The Case $\varepsilon=0$. Some existence And nonexistence Results FOR THE GENERAL CASE}

First, we introduce some notations. Fix $\nu>0$ and consider the Banach spaces

$$
U:=C_{0}^{2+\nu}(\bar{\Omega}), \quad V:=C^{\nu}(\bar{\Omega})
$$

ordered by their cones of nonnegative functions $P_{U}$ and $P_{V}$, respectively. The interior of a cone $P$ will be denoted by $\dot{P}$ and we shall write $f \geq g$ if $f-g \in P$, $f>g$ if $f-g \in P \backslash\{0\}$ and $f \gg g$ if $f-g \in \dot{P}$. The interior of $P_{U}$ is the set of $w \in U$ such that $w(x)>0$ for all $x \in \Omega$ and $\frac{\partial w}{\partial n}(x)<0$ for all $x \in \partial \Omega$, where $n$ stands for the outward unit normal at $\partial \Omega$. The parabolic maximum principle ensures that if $u$ is a solution of (1.1) with initial data $u_{0}>0$, then $u \in \dot{P}_{U}$ for any $t>0$.

Given $q \in C(\bar{\Omega})$ and a smooth subdomain $\Omega_{1} \subset \Omega$ we consider the linear elliptic eigenvalue problem

$$
(\mathcal{L}+q(x)) \varphi=\sigma \varphi \quad \text { in } \Omega_{1}, \quad v=0 \quad \text { on } \partial \Omega_{1} .
$$

This problem possesses a unique eigenvalue to a positive eigenfunction, denoted by

$$
\sigma_{1}^{\Omega_{1}}[\mathcal{L}+q] \text { and } \varphi_{1}^{\Omega_{1}}[\mathcal{L}+q]
$$

respectively. By a principal eigenvalue we mean an eigenvalue to a positive eigenfunction. This result can be obtained from the strong maximum principle and the Krein-Rutman theorem, [19]. The principal eigenvalue is real and algebraically simple. Moreover, the principal eigenfunction lies in the interior of the positive cone of $U$ and the next characterization of the strong maximum principle going back to 21] and [18] holds (cf. 19]).

Theorem 2.1. The following conditions are equivalent:

(i) $\sigma_{1}^{\Omega_{1}}[\mathcal{L}+q]>0$.

(ii) There exists $\psi \in C^{1}\left(\bar{\Omega}_{1}\right) \cap C^{2}\left(\Omega_{1}\right)$ such that $\psi(x)>0$ for all $x \in \Omega_{1}$ and

$$
(\mathcal{L}+q(x)) \psi \geq 0 \quad \text { in } \quad \Omega_{1}, \quad \psi \geq 0 \quad \text { on } \partial \Omega_{1},
$$

with some of these inequalities strict. In other words, the problem

$$
(\mathcal{L}+q(x)) w=0 \quad \text { in } \quad \Omega_{1}, \quad w=0 \quad \text { on } \partial \Omega_{1},
$$

possesses a strict positive supersolution. In this case, it will be simply said that $\mathcal{L}+q$ possesses a strict positive supersolution.

(iii) The operator $\mathcal{L}+q$ under Dirichlet conditions satisfies the strong maximum principle, i.e., if $w \in C^{1}\left(\bar{\Omega}_{1}\right) \cap C^{2}\left(\Omega_{1}\right)$ satisfies

$$
(\mathcal{L}+q) w \geq 0 \quad \text { in } \quad \Omega_{1} \quad w \geq 0 \quad \text { on } \partial \Omega_{1},
$$

then $w \geq 0$ in $\Omega_{1}$. Moreover, if some of these inequalities is strict, then $w \gg 0$.

The equivalence of assertions (i), (iii) in the statement of Theorem 2.1 was shown to occur for very general classes of second order elliptic operators in general nonsmooth domains by Beresticky, Nirenberg and Varadhan [6], but in this work nothing was said about the equivalence between the maximum principle and the existence of a strict positive supersolution, being one of the main features which will be used throughout the rest of this paper. 
Theorem 2.1 is substantially sharper than the generalized maximum principle of Protter and Weinberger [25]. The generalized maximum principle of [25] shows that the existence of a positive supersolution positive on $\partial \Omega_{1}$ is sufficient for the strong maximum principle, while Theorem 2.1 characterizes the strong maximum principle in terms of the existence of a strict positive supersolution not necessarily positive on the boundary of the domain. We should point out that Theorem 2.1 is valid for general second order elliptic operators not necessarily selfadjoint (cf. [16]).

The following consequence of the strong maximum principle will be very useful later.

Theorem 2.2. (i) If there exists $u>0$ in $\Omega_{1}$ such that $\left.u\right|_{\partial \Omega_{1}}=0$ and $(\mathcal{L}+q) u>0$ in $\Omega_{1}$, then

$$
\sigma_{1}^{\Omega_{1}}[\mathcal{L}+q]>0 .
$$

(ii) If there exists $u>0$ in $\Omega_{1}$ such that $\left.u\right|_{\partial \Omega_{1}}=0$ and $(\mathcal{L}+q) u<0$ in $\Omega_{1}$, then

$$
\sigma_{1}^{\Omega_{1}}[\mathcal{L}+q]<0 .
$$

Proof. By Theorem 2.1, part (i) is already known. Moreover, if there exists $u>0$ in $\Omega_{1}$ such that $\left.u\right|_{\partial \Omega_{1}}=0$ and $(\mathcal{L}+q) u<0$ in $\Omega_{1}$, then $\sigma_{1}^{\Omega_{1}}[\mathcal{L}+q] \leq 0$. By Theorem 3.2 (iv) of Amann [2], this eigenvalue cannot vanish. This completes the proof.

By the strong maximum principle, the analytic semigroup $e^{t(\mathcal{L}+q)}, t \geq 0$, is strongly order preserving and hence the principal eigenvalue is strictly dominant in the sense of [17], i.e. for any other eigenvalue $\sigma \in \mathbb{C}$ of $(2.1)$

$$
\operatorname{Re} \sigma>\sigma_{1}^{\Omega_{1}}[\mathcal{L}+q] .
$$

Thus, the sign of $\sigma_{1}^{\Omega_{1}}[\mathcal{L}+q]$ provides us with the stability properties of the zero solution of

$$
\left(\partial_{t}+\mathcal{L}+q(x)\right) u=0 \quad \text { in } \Omega_{1} \times(0, \infty),\left.\quad u\right|_{\partial \Omega_{1} \times(0, \infty)}=0 .
$$

The zero solution is exponentially asymptotically stable if $\sigma_{1}^{\Omega_{1}}[\mathcal{L}+q]>0$, and unstable if $\sigma_{1}^{\Omega_{1}}[\mathcal{L}+q]<0$. Furthermore, the linearized stability principle is satisfied and therefore the zero solution of (1.1) is exponentially asymptotically stable if $\lambda<\sigma_{1}^{\Omega}[\mathcal{L}]$, and unstable if $\lambda>\sigma_{1}^{\Omega}[\mathcal{L}]$.

Theorem 2.1 can be used to get the standard comparison properties of principal eigenvalues (cf. [18]) and it provides us with a necessary condition for the existence of positive solutions.

Theorem 2.3. Suppose that (1.3) possesses a positive solution. Then,

$$
\lambda<\sigma_{1}^{\Omega_{0} \cup \Omega_{-}}[\mathcal{L}] \quad\left(\Omega_{0} \cup \Omega_{-}=\Omega \backslash \bar{\Omega}_{+}\right) .
$$

Proof. Let $u$ be a positive solution of (1.3). In the region $\Omega_{0} \cup \Omega_{-}$we have

$$
a_{\varepsilon} u=-\varepsilon a^{-} u \leq 0
$$

and hence

$$
(\mathcal{L}-\lambda) u \geq 0 \quad \text { in } \Omega_{0} \cup \Omega_{-} .
$$

Moreover, $u=0$ on $\partial \Omega, u>0$ on $\partial \Omega_{+}$, and $\partial\left(\Omega_{0} \cup \Omega_{-}\right)=\partial \Omega \cup \partial \Omega_{+}$. Thus, $u$ is a strict positive supersolution of $\mathcal{L}-\lambda$ in $\Omega_{0} \cup \Omega_{-}$and therefore it follows from Theorem 2.1 that

$$
\sigma_{1}^{\Omega_{0} \cup \Omega_{-}}[\mathcal{L}-\lambda]>0 .
$$

This completes the proof. 
When $\varepsilon=0,(2.2)$ provides us with an optimal upper bound of the range of $\lambda$ 's for which (1.3) admits a positive solution. The following result characterizes the existence in this special case.

Theorem 2.4. Assume $\varepsilon=0$. Then, (1.3) possesses a positive solution if, and only if,

$$
\sigma_{1}^{\Omega}[\mathcal{L}]<\lambda<\sigma_{1}^{\Omega \backslash \bar{\Omega}_{+}}[\mathcal{L}]
$$

Moreover, the positive solution is unique if it exists and if we denote it by $\theta_{\lambda}$, then the mapping $\lambda \rightarrow \theta_{\lambda}$ is increasing and

$$
\lim _{\lambda \downarrow \sigma_{1}^{\Omega}[\mathcal{L}]}\left\|\theta_{\lambda}\right\|_{\infty}=0, \quad \lim _{\left.\lambda \uparrow \sigma_{1}^{\Omega \backslash \bar{\Omega}_{+}}+\mathcal{L}\right]} \theta_{\lambda}=\infty
$$

uniformly on compact subsets of $\Omega \backslash \bar{\Omega}_{+}$.

Furthermore, 0 is a global attractor for (1.1) if $\lambda \leq \sigma_{1}^{\Omega}[\mathcal{L}], \theta_{\lambda}$ is a global attractor of the positive solutions of (1.1) if (2.3) is satisfied, and

$$
\lim _{t \uparrow \infty}\left\|u\left(\cdot, t ; u_{0}\right)\right\|_{\infty}=\infty
$$

if $u_{0}>0$ and $\lambda \geq \sigma_{1}^{\Omega \backslash \bar{\Omega}_{+}}[\mathcal{L}]$.

Except for the uniform convergence of the solution to infinity as $\lambda \uparrow \sigma_{1}^{\Omega \backslash \Omega_{+}}[\mathcal{L}]$, this result goes back to [7, 11], [14] and [22]. References 7], [11] and [22] dealt with the special case when $\mathcal{L}=-\Delta$, while [14] dealt with general second order elliptic operators not necessarily selfadjoint. In [7], Brezis and Oswald found the characterization of the existence as well as the uniqueness by means of variational methods. Independently, Ouyang [22] used some global continuation methods to show the existence and the uniqueness as well as the divergence of the $L^{2}$-norm of the positive solution as $\lambda \uparrow \sigma_{1}^{\Omega \backslash \bar{\Omega}_{+}}$, and Del Pino [11] gave a simpler variational proof of Ouyang's result including the limiting bahaviour of the solution. Fraile et al. 14] used monotonicity methods to show the existence, the uniqueness and the global attractive character of the positive solution for general second order elliptic operators, where variational methods do not work. Here, we will give a new and shorter proof of the existence which works out as well for general operators not necessarily selfadjoint.

Proof of Theorem 2.4. Set $D_{0}:=\Omega \backslash \bar{\Omega}_{+}$and suppose that (1.3) possesses a positive solution. Due to Theorem 2.3, $\lambda<\sigma_{1}^{D_{0}}[\mathcal{L}]$. Moreover, in $\Omega$ we have that

$$
(\mathcal{L}-\lambda) u=a_{0} u>0
$$

and hence we find from Theorem 2.2(i) that $\lambda<\sigma_{1}^{\Omega}[\mathcal{L}]$. Therefore, $(2.3)$ is necessary for the existence of a positive solution. To show the sufficiency of $(2.3)$ we use the method of sub and supersolutions. Assume (2.3) and let $\varphi$ denote the principal eigenfunction associated with $\sigma_{1}^{\Omega}[\mathcal{L}]$. Then, $\delta \varphi$ is a positive subsolution of $(1.3)$ if $\delta>0$ is sufficiently small. So, it suffices to construct a supersolution in the interior of the positive cone. For $\delta>0$ small set

$$
D_{\delta}:=D_{0}+B_{\delta},
$$

where $B_{\delta}$ is the ball of radius $\delta$ centered at 0 . Let $\varphi_{\delta}$ denote the principal eigenfunction associated with $\sigma_{1}^{D_{\delta}}[\mathcal{L}]$. Let $\psi_{\delta}$ be any smooth positive function such that $\psi_{\delta}=\varphi_{\delta}$ in $D_{\frac{\delta}{2}}$ and $\psi_{\delta}$ is bounded away from zero uniformly in the complement of 
$D_{\frac{\delta}{2}}$. Since $\lambda<\sigma_{1}^{D_{0}}[\mathcal{L}]$, the continuous dependence of the principal eigenvalue with respect to the domain shows that

$$
\lambda<\sigma_{1}^{D_{\delta}}[\mathcal{L}]
$$

if $\delta>0$ is sufficiently small [19]. Choose $\delta$ satisfying (2.5). We claim that $\kappa \psi_{\delta}$ is a supersolution of (1.3) if $\kappa>1$ is sufficiently large. Indeed, in the complement of $D_{\frac{\delta}{2}}$ we have that $a_{0} \psi_{\delta}$ is positive and bounded away from zero. Hence,

$$
\mathcal{L} \psi_{\delta} \geq \lambda \psi_{\delta}-a \kappa \psi_{\delta}^{2}
$$

for large $\kappa$. In $D_{\frac{\delta}{2}}, \psi_{\delta}=\varphi_{\delta}$ and hence (2.5) implies

$$
\mathcal{L}\left(\kappa \psi_{\delta}\right)=\kappa \sigma_{1}^{D_{\delta}}[\mathcal{L}] \psi_{\delta}>\lambda \kappa \psi_{\delta} \geq \lambda \kappa \psi_{\delta}-a_{0}\left(\kappa \psi_{\delta}\right)^{2} .
$$

This completes the proof of the existence. For a proof of the uniqueness, the asymptotic behaviour of the nonnegative solutions and the validity of the first limit of (2.4) we refer to [14].

We now show the validity of the second limit of (2.4). Assume (2.3) and let $\theta_{\lambda}$ denote the positive solution of (1.3). Then, (1.3) implies

$$
\left(\mathcal{L}-\lambda+a_{0} \theta_{\lambda}\right) \theta_{\lambda}=0
$$

and hence, we find from the uniqueness of the principal eigenvalue that

$$
\sigma_{1}^{\Omega}\left[\mathcal{L}-\lambda+a_{0} \theta_{\lambda}\right]=0 .
$$

Differentiating (2.6) with respect to $\lambda$ yields

$$
\left(\mathcal{L}-\lambda+2 a_{0} \theta_{\lambda}\right) \frac{d \theta_{\lambda}}{d \lambda}=\theta_{\lambda} .
$$

Moreover, (2.7) implies

$$
\sigma_{1}^{\Omega}\left[\mathcal{L}-\lambda+2 a_{0} \theta_{\lambda}\right]>0
$$

and hence it follows from (2.8) that

$$
\frac{d \theta_{\lambda}}{d \lambda}=\left(\mathcal{L}-\lambda+2 a_{0} \theta_{\lambda}\right)^{-1} \theta_{\lambda} \gg 0 .
$$

This shows that $\lambda \rightarrow \theta_{\lambda}$ is increasing. Moreover, if we fix

$$
\lambda_{1} \in\left(\sigma_{1}^{\Omega}[\mathcal{L}], \sigma_{1}^{D_{0}}[\mathcal{L}]\right),
$$

then we find from (2.8) that

$$
\left(\mathcal{L}-\lambda+2 a_{0} \theta_{\lambda}\right) \frac{d \theta_{\lambda}}{d \lambda} \gg \theta_{\lambda_{1}}, \quad \forall \lambda>\lambda_{1} .
$$

Let $\varphi_{0} \gg 0$ denote the principal eigenfunction associated with $\sigma_{1}^{D_{0}}[\mathcal{L}]$. Let $c>0$ be a constant such that $\theta_{\lambda_{1}}>c \varphi_{0}$. Then, (2.10) gives

$$
\left(\mathcal{L}-\lambda+2 a_{0} \theta_{\lambda}\right) \frac{d \theta_{\lambda}}{d \lambda} \gg c \varphi_{0}, \quad \forall \lambda>\lambda_{1} .
$$

Moreover, $\theta_{\lambda}>0=c \varphi_{0}$ on $\partial D_{0}$ and $a_{0}=0$ in $D_{0}$. Thus, it follows from the strong maximum principle that

$$
\frac{d \theta_{\lambda}}{d \lambda} \gg \Theta_{\lambda}
$$

where $\Theta_{\lambda}$ is the solution of

$$
(\mathcal{L}-\lambda) \Theta_{\lambda}=c \varphi_{0}
$$


in $D_{0}$ under homogeneous Dirichlet boundary conditions. By the definition of $\varphi_{0}$ we have that

$$
\Theta_{\lambda}=\frac{c}{\sigma_{1}^{D_{0}}[\mathcal{L}]-\lambda} \varphi_{0}
$$

This completes the proof, since this function grows to infinity as $\lambda \uparrow \sigma_{1}^{D_{0}}[\mathcal{L}]$ uniformly on compact subsets of $D_{0}$.

Theorem 2.4 provides us with the following result.

Theorem 2.5. Set

$$
D_{0}:=\Omega \backslash \bar{\Omega}_{+}
$$

and take

$$
\delta \in\left(0, \sigma_{1}^{D_{0}}[\mathcal{L}]-\sigma_{1}^{\Omega}[\mathcal{L}]\right) .
$$

Then, there exists $\varepsilon(\delta)>0$ such that (1.3) possesses a positive solution for each

$$
\lambda \in\left(\sigma_{1}^{\Omega}[\mathcal{L}], \sigma_{1}^{D_{0}}[\mathcal{L}]-\delta\right]
$$

provided $\varepsilon \in(0, \varepsilon(\delta))$.

Proof. Set

$$
\lambda_{1}:=\sigma_{1}^{D_{0}}[\mathcal{L}]-\delta .
$$

Thanks to Theorem 2.4, (1.3) possesses a unique positive solution if $(\lambda, \varepsilon)=\left(\lambda_{1}, 0\right)$. Namely, $\theta_{\lambda_{1}}$. Consider the operator $\mathcal{N}: U \times[0, \infty) \rightarrow V$ defined by

$$
\mathcal{N}(u, \varepsilon)=\mathcal{L} u-\lambda_{1} u+a_{\varepsilon} u^{2},
$$

whose zeros are the solutions of (1.3) for $\lambda=\lambda_{1}$. We have $\mathcal{N}\left(\theta_{\lambda_{1}}, 0\right)=0$. Moreover, due to $(2.9)$,

$$
D_{u} \mathcal{N}\left(\theta_{\lambda_{1}}, 0\right)=\mathcal{L}-\lambda_{1}+2 a_{0} \theta_{\lambda_{1}}
$$

is a topological isomorphism. Therefore, the implicit function theorem guarantees that there exist $\varepsilon(\delta)>0$ and an analytic mapping $u(\cdot):[0, \varepsilon(\delta)] \rightarrow U$ such that $u(0)=\theta_{\lambda_{1}}$ and $\mathcal{N}(u(\varepsilon), \varepsilon)=0$ for each $\varepsilon \in[0, \varepsilon(\delta)]$. In fact, since $\theta_{\lambda_{1}} \gg 0$, $u(\varepsilon) \gg 0$ provided $\varepsilon$ is sufficiently small, which can be accomplished by reducing $\varepsilon(\delta)$, if necessary.

Assume $\lambda \in\left(\sigma_{1}^{\Omega}[\mathcal{L}], \lambda_{1}\right]$ and $\varepsilon \in[0, \varepsilon(\delta)]$. Then,

$$
\begin{aligned}
\mathcal{L} u(\varepsilon(\delta)) & =\lambda_{1} u(\varepsilon(\delta))-\left(a^{+}-\varepsilon(\delta) a^{-}\right) u^{2}(\varepsilon(\delta)) \\
& \geq \lambda u(\varepsilon(\delta))-\left(a^{+}-\varepsilon a^{-}\right) u^{2}(\varepsilon(\delta))
\end{aligned}
$$

and hence $u(\varepsilon(\delta))$ is a positive supersolution of (1.3). Moreover, if we denote by $\varphi_{1}$ the principal eigenfunction associated with $\sigma_{1}^{\Omega}[\mathcal{L}]$, then $c \varphi_{1}$ is a subsolution of (1.3) for $c$ sufficiently small. This completes the proof.

The next result shows the optimality of Theorem 2.5.

Theorem 2.6. Assume that

$$
\sigma_{1}^{\Omega}[\mathcal{L}]<\lambda<\sigma_{1}^{\Omega \backslash \bar{\Omega}_{+}}[\mathcal{L}]
$$


and that (1.3) possesses a positive solution. Then,

$$
\varepsilon \leq \inf _{\delta>0} \frac{\sigma_{1}^{\Omega_{-}^{\delta}}[\mathcal{L}]-\lambda}{\min _{\Omega_{-}^{\delta}} a^{-} \min _{\Omega_{-}^{\delta}} \theta_{\lambda}},
$$

where

$$
\Omega_{-}^{\delta}:=\left\{x \in \Omega_{-}: d\left(x, \partial \Omega_{-}\right)>\delta\right\}
$$

and $\theta_{\lambda}$ is the unique positive solution of (1.3) for $\varepsilon=0$. In particular, there exists

$$
\lambda^{*}(\varepsilon)<\sigma_{1}^{\Omega \backslash \bar{\Omega}_{+}}[\mathcal{L}]
$$

such that (1.3) does not admit a positive solution if $\lambda \geq \lambda^{*}(\varepsilon)$.

Remark 2.7. Since $\Omega_{-}^{\delta} \subset \Omega \backslash \bar{\Omega}_{+},(2.4)$ implies

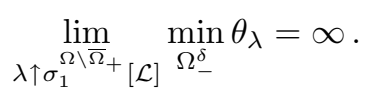

Therefore, the right-hand side of $(2.12)$ goes to zero as $\lambda \uparrow \sigma_{1}^{\Omega \backslash \bar{\Omega}_{+}}[\mathcal{L}]$. Similarly,

$$
\lim _{\lambda \downarrow \sigma_{1}^{\Omega}[\mathcal{L}]} \min _{\Omega_{-}^{\delta}} \theta_{\lambda}=0,
$$

and therefore, the right-hand side of $(2.12)$ grows to infinity as $\lambda \downarrow \sigma_{1}^{\Omega}[\mathcal{L}]$. These results are rather natural. If we want (1.3) to have positive solutions for $\lambda$ close to $\sigma_{1}^{\Omega \backslash \bar{\Omega}_{+}}[\mathcal{L}]$, then we should take $\varepsilon$ small enough. On the contrary, if we are looking for positive solutions when $\lambda$ is close to $\sigma_{1}^{\Omega}[\mathcal{L}]$, then we can allow $\varepsilon$ to be very large and this is so because for any $\varepsilon,(u, \lambda)=\left(0, \sigma_{1}^{\Omega}[\mathcal{L}]\right)$ is a bifurcation point to positive solutions. The nature of this bifurcation will be analyzed in Section 3.

Proof of Theorem 2.6. Let $u$ be a positive solution of (1.3). Then, (1.3) gives

$$
0=\sigma_{1}^{\Omega}\left[\mathcal{L}-\lambda+a_{\varepsilon} u\right]<\sigma_{1}^{\Omega_{-}^{\delta}}\left[\mathcal{L}-\lambda+a_{\varepsilon} u\right]=\sigma_{1}^{\Omega_{-}^{\delta}}\left[\mathcal{L}-\lambda-\varepsilon a^{-} u\right]
$$

and hence

$$
0<\sigma_{1}^{\Omega_{-}^{\delta}}[\mathcal{L}]-\lambda-\varepsilon \min _{\Omega_{-}^{\delta}} a^{-} \min _{\Omega_{-}^{\delta}} u
$$

Moreover,

$$
\mathcal{L} u=\lambda u-a_{\varepsilon} u^{2}>\lambda u-a_{0} u^{2}
$$

and so $u$ is a positive supersolution of (1.3) for $\varepsilon=0$. By the uniqueness, $u \geq \theta_{\lambda}$. Finally, by substituting this estimate into (2.13) we get (2.12). This completes the proof.

\section{LOCAL BIFURCATION ANALYSIS AND SOME GLOBAL EXISTENCE RESULTS}

The solutions of (1.3) are the zeros of the operator $\mathcal{F}: U \times \mathbb{R}^{2} \rightarrow V$ defined by

$$
\mathcal{F}(u, \lambda, \varepsilon)=\mathcal{L} u-\lambda u+a_{\varepsilon}(x) u^{2}, \quad(u, \lambda, \varepsilon) \in U \times \mathbb{R}^{2} .
$$

Let $\varphi_{1} \gg 0$ and $Z$ denote the principal eigenfunction associated with $\sigma_{1}^{\Omega}[\mathcal{L}]$ and the $L^{2}$-orthogonal of $\varphi_{1}$ in $U$, respectively. For any $(v, \lambda, \varepsilon, s) \in Z \times \mathbb{R}^{3}$ define

$$
\mathcal{G}(v, \lambda, \varepsilon, s)=\mathcal{L}\left(\varphi_{1}+v\right)-\lambda\left(\varphi_{1}+v\right)+s a_{\varepsilon}(x)\left(\varphi_{1}+v\right)^{2} .
$$


The operators $\mathcal{F}$ and $\mathcal{G}$ are analytic in all their arguments. Moreover, $\mathcal{G}(v, \lambda, \varepsilon, s)=$ $s^{-1} \mathcal{F}\left(s\left(\varphi_{1}+v\right), \lambda, \varepsilon\right)$ if $s \neq 0$ and $\mathcal{G}(v, \lambda, \varepsilon, 0)=D_{u} \mathcal{F}(0, \lambda, \varepsilon)\left(\varphi_{1}+v\right)$. Hence, the zeros of $\mathcal{G}$ provide us with zeros of $\mathcal{F}$. Fix $\varepsilon=\varepsilon_{0}$ arbitrary. Then,

$$
\mathcal{G}\left(0, \sigma_{1}^{\Omega}[\mathcal{L}], \varepsilon_{0}, 0\right)=0
$$

and its Fréchèt differential

$$
D_{(v, \lambda)} \mathcal{G}\left(0, \sigma_{1}^{\Omega}[\mathcal{L}], \varepsilon_{0}, 0\right): Z \times \mathbb{R} \rightarrow V
$$

is given by

$$
D_{(v, \lambda)} \mathcal{G}\left(0, \sigma_{1}^{\Omega}[\mathcal{L}], \varepsilon_{0}, 0\right)(v, \lambda)=\left(\mathcal{L}-\sigma_{1}^{\Omega}[\mathcal{L}]\right) v-\lambda \varphi_{1} .
$$

Since $\varphi_{1} \notin R\left(\mathcal{L}-\sigma_{1}^{\Omega}[\mathcal{L}]\right)$, the open mapping theorem implies that this operator is an isomorphism and therefore the implicit function theorem gives the following result.

Proposition 3.1. There exist $\delta_{1}>0, \delta_{2}>0$ and two analytic mappings

$$
v:\left(\varepsilon_{0}-\delta_{1}, \varepsilon_{0}+\delta_{1}\right) \times\left(-\delta_{2}, \delta_{2}\right) \rightarrow Z, \quad \lambda:\left(\varepsilon_{0}-\delta_{1}, \varepsilon_{0}+\delta_{1}\right) \times\left(-\delta_{2}, \delta_{2}\right) \rightarrow \mathbb{R},
$$

such that

$$
v\left(\varepsilon_{0}, 0\right)=0, \quad \lambda\left(\varepsilon_{0}, 0\right)=\sigma_{1}^{\Omega}[\mathcal{L}]
$$

and for each $(\varepsilon, s) \in\left(\varepsilon_{0}-\delta_{1}, \varepsilon_{0}+\delta_{1}\right) \times\left(-\delta_{2}, \delta_{2}\right)$

$$
\mathcal{G}(v(\varepsilon, s), \lambda(\varepsilon, s), \varepsilon, s)=0 .
$$

Moreover, these are the unique zeros of $\mathcal{G}$ in a neighborhood of $(v, \lambda, \varepsilon, s)=$ $\left(0, \sigma_{1}^{\Omega}[\mathcal{L}], \varepsilon_{0}, 0\right)$.

Remark 3.2. For all $\varepsilon \in \mathbb{R}$ we have

$$
\mathcal{G}\left(0, \sigma_{1}^{\Omega}[\mathcal{L}], \varepsilon, 0\right)=0
$$

and hence, by the uniqueness of Proposition 3.1,

$$
v(\varepsilon, 0)=0, \quad \lambda(\varepsilon, 0)=\sigma_{1}^{\Omega}[\mathcal{L}]
$$

for all $\varepsilon \in\left(\varepsilon_{0}-\delta_{1}, \varepsilon_{0}+\delta_{1}\right)$. Thus, due to (3.3), for each $(\varepsilon, s) \in\left(\varepsilon_{0}-\delta_{1}, \varepsilon_{0}+\delta_{1}\right) \times$ $\left(-\delta_{2}, \delta_{2}\right)$ we have

$$
\mathcal{F}\left(s\left[\varphi_{1}+v(\varepsilon, s)\right], \lambda(\varepsilon, s)\right)=0 .
$$

Therefore, for each $\varepsilon \in\left(\varepsilon_{0}-\delta_{1}, \varepsilon_{0}+\delta_{1}\right)$ there is a curve of nonzero solutions of (1.3) emanating from $(u, \lambda)=(0, \lambda)$ at $\lambda=\sigma_{1}^{\Omega}[\mathcal{L}]$. These solutions are positive if $s>0$ and negative if $s<0$.

The celebrated theorem of Crandall and Rabinowitz [8] shows that any nontrivial solution $(u, \lambda)$ of $(1.3)$ in a neighborhood of $(u, \lambda)=\left(0, \sigma_{1}^{\Omega}[\mathcal{L}]\right)$ must be of the form

$$
(u, \lambda)=\left(s\left[\varphi_{1}+v(\varepsilon, s)\right], \lambda(\varepsilon, s)\right)
$$

for some $s \simeq 0, s \neq 0$. The next result shows that the neighborhood can be chosen to be independent of $\varepsilon \simeq \varepsilon_{0}$. A proof of it can be found in [13.

Proposition 3.3. There exists a neighborhood $\mathcal{B}$ of $(u, \lambda, \varepsilon)=\left(0, \sigma_{1}^{\Omega}[\mathcal{L}], \varepsilon_{0}\right)$ in $U \times \mathbb{R}^{2}$ such that if $(u, \lambda, \varepsilon) \in \mathcal{B}, u \neq 0$ and $\mathcal{F}(u, \lambda, \varepsilon)=0$, then $(u, \lambda)=$ $\left(s\left[\varphi_{1}+v(\varepsilon, s)\right], \lambda(\varepsilon, s)\right)$ for some $s \neq 0$. 

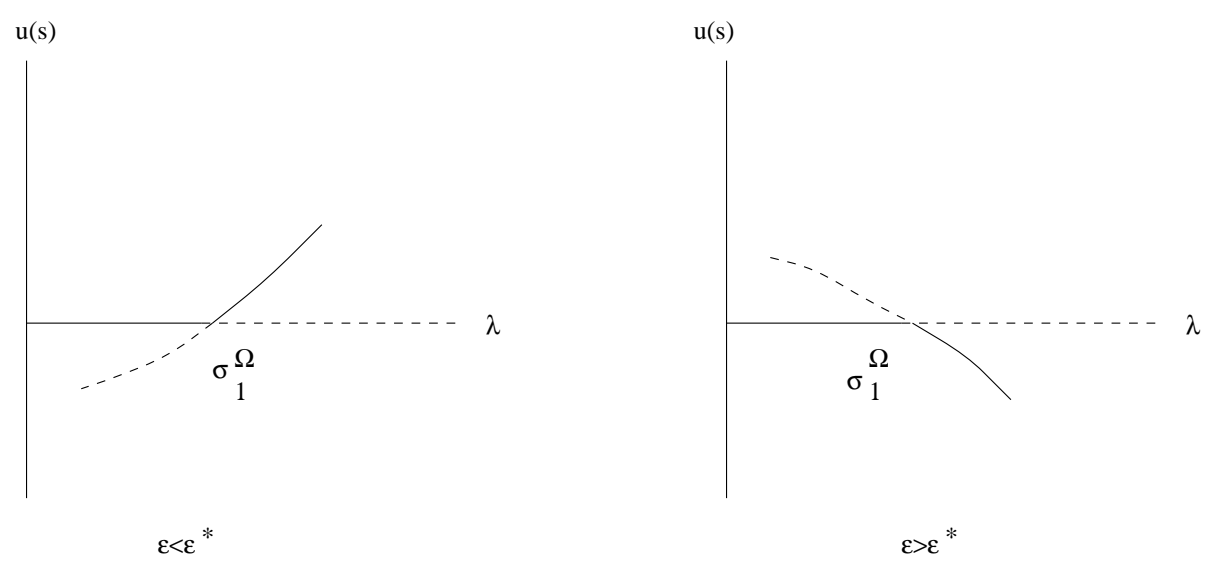

FiguRE 1. Local bifurcation diagrams.

The global theorem of Rabinowitz [26] shows that the continuum $\mathcal{C}^{+}$of positive solutions of (1.3) emanating from $u=0$ at $\lambda=\sigma_{1}^{\Omega}[\mathcal{L}]$ is unbounded in $U \times \mathbb{R}$.

We now analyze the nature of the local bifurcation. Differentiating (3.3) with respect to $s$ we find that

$$
\begin{aligned}
D_{v} \mathcal{G}(v(\varepsilon, s), \lambda(\varepsilon, s), \varepsilon, s) \frac{d v}{d s}(\varepsilon, s) & +D_{\lambda} \mathcal{G}(v(\varepsilon, s), \lambda(\varepsilon, s), \varepsilon, s) \frac{d \lambda}{d s}(\varepsilon, s) \\
& +D_{s} \mathcal{G}(v(\varepsilon, s), \lambda(\varepsilon, s), \varepsilon, s)=0 .
\end{aligned}
$$

Specifying (3.6) at $s=0$ and using (3.4) gives

$$
D_{v} \mathcal{G}\left(0, \sigma_{1}^{\Omega}[\mathcal{L}], \varepsilon, 0\right) \frac{d v}{d s}(\varepsilon, 0)+D_{\lambda} \mathcal{G}\left(0, \sigma_{1}^{\Omega}[\mathcal{L}], \varepsilon, 0\right) \frac{d \lambda}{d s}(\varepsilon, 0)+a_{\varepsilon}(x) \varphi_{1}^{2}=0,
$$

which can be written in the form

$$
\left(\mathcal{L}-\sigma_{1}^{\Omega}[\mathcal{L}]\right) \frac{d v}{d s}(\varepsilon, 0)-\frac{d \lambda}{d s}(\varepsilon, 0) \varphi_{1}+a_{\varepsilon}(x) \varphi_{1}^{2}=0 .
$$

Multiplying (3.7) by $\varphi_{1}$, integrating in $\Omega$ and normalizing $\varphi_{1}$ so that $\int_{\Omega} \varphi_{1}^{2}=1$, we find that

$$
\frac{d \lambda}{d s}(\varepsilon, 0)=\int_{\Omega} a_{\varepsilon} \varphi_{1}^{3}=\int_{\Omega_{+}} a^{+} \varphi_{1}^{3}-\varepsilon \int_{\Omega_{-}} a^{-} \varphi_{1}^{3}
$$

Setting

$$
\varepsilon^{*}:=\frac{\int_{\Omega_{+}} a^{+} \varphi_{1}^{3}}{\int_{\Omega_{-}} a^{-} \varphi_{1}^{3}} .
$$

(3.8) implies $\frac{d \lambda}{d s}\left(\varepsilon^{*}, 0\right)=0$. Moreover, $\frac{d \lambda}{d s}(\varepsilon, 0)<0$ if $\varepsilon>\varepsilon^{*}$, and $\frac{d \lambda}{d s}(\varepsilon, 0)>0$ if $\varepsilon<\varepsilon^{*}$. Thus, the bifurcation is transcritical if $\varepsilon \neq \varepsilon^{*}$. Moreover, the positive solutions emanate supercritically if $\varepsilon<\varepsilon^{*}$ and subcritically if $\varepsilon>\varepsilon^{*}$. Figure 1 shows the corresponding local bifurcation diagrams.

By using the exchange stability principle [9], the following result holds.

Theorem 3.4. (i) If $\varepsilon>\varepsilon^{*}$, then there exists $\lambda_{*}<\sigma_{1}^{\Omega}[\mathcal{L}]$ such that (1.3) possesses an unstable positive solution for each $\lambda \in\left[\lambda^{*}, \sigma_{1}^{\Omega}[\mathcal{L}]\right)$.

(i) If $\varepsilon<\varepsilon^{*}$, then there exists $\lambda^{*}>\sigma_{1}^{\Omega}[\mathcal{L}]$ such that (1.3) possesses a stable positive solution for each $\lambda \in\left(\sigma_{1}^{\Omega}[\mathcal{L}], \lambda^{*}\right]$. 
Now, we analyze the nature of the local bifurcation when $\varepsilon=\varepsilon^{*}$. In this case,

$$
\frac{d \lambda}{d s}\left(\varepsilon^{*}, 0\right)=0
$$

and so higher order terms are needed. Differentiating (3.6) with respect to $s$ gives

$$
\begin{aligned}
D_{v v} \mathcal{G}(s)[ & \left.\frac{d v}{d s}\left(\varepsilon^{*}, s\right), \frac{d v}{d s}\left(\varepsilon^{*}, s\right)\right]+\frac{d \lambda}{d s}\left(\varepsilon^{*}, s\right) D_{v \lambda} \mathcal{G}(s) \frac{d v}{d s}\left(\varepsilon^{*}, s\right)+D_{v s} \mathcal{G}(s) \frac{d v}{d s}\left(\varepsilon^{*}, s\right) \\
& +D_{v} \mathcal{G}(s) \frac{d^{2} v}{d s^{2}}\left(\varepsilon^{*}, s\right)+\frac{d \lambda}{d s}\left(\varepsilon^{*}, s\right) D_{\lambda v} \mathcal{G}(s) \frac{d v}{d s}\left(\varepsilon^{*}, s\right)+\left(\frac{d \lambda}{d s}\left(\varepsilon^{*}, s\right)\right)^{2} D_{\lambda \lambda} \mathcal{G}(s) \\
& +\frac{d \lambda}{d s}\left(\varepsilon^{*}, s\right) D_{\lambda s} \mathcal{G}(s)+\frac{d^{2} \lambda}{d s^{2}}\left(\varepsilon^{*}, s\right) D_{\lambda} \mathcal{G}(s)+D_{s v} \mathcal{G}(s) \frac{d v}{d s}\left(\varepsilon^{*}, s\right) \\
& +\frac{d \lambda}{d s}\left(\varepsilon^{*}, s\right) D_{s \lambda} \mathcal{G}(s)+D_{s s} \mathcal{G}(s)=0,
\end{aligned}
$$

where, in order to shorten the notations, we have denoted

$$
(s):=\left(v\left(\varepsilon^{*}, s\right), \lambda\left(\varepsilon^{*}, s\right), \varepsilon^{*}, s\right) \text {. }
$$

We already know that

$$
v\left(\varepsilon^{*}, 0\right)=0, \quad \lambda\left(\varepsilon^{*}, 0\right)=\sigma_{1}^{\Omega}[\mathcal{L}], \quad \frac{d \lambda}{d s}\left(\varepsilon^{*}, 0\right)=0 .
$$

Moreover, (3.2) implies

$$
\begin{gathered}
D_{v v} \mathcal{G}\left(0, \sigma_{1}^{\Omega}[\mathcal{L}], \varepsilon^{*}, 0\right)=0, \quad D_{v s} \mathcal{G}\left(0, \sigma_{1}^{\Omega}[\mathcal{L}], \varepsilon^{*}, 0\right)=2 a_{\varepsilon} \varphi_{1}, \\
D_{s s} \mathcal{G}\left(0, \sigma_{1}^{\Omega}[\mathcal{L}], \varepsilon^{*}, 0\right)=0, \\
D_{v} \mathcal{G}\left(0, \sigma_{1}^{\Omega}[\mathcal{L}], \varepsilon^{*}, 0\right)=\mathcal{L}-\sigma_{1}^{\Omega}[\mathcal{L}], \quad D_{\lambda} \mathcal{G}\left(0, \sigma_{1}^{\Omega}[\mathcal{L}], \varepsilon^{*}, 0\right)=-\varphi_{1} .
\end{gathered}
$$

Therefore, specifying (3.11) at $s=0$ and substituting all these relations into the resulting equation, we find that

$$
\left(\mathcal{L}-\sigma_{1}^{\Omega}[\mathcal{L}]\right) \frac{d^{2} v}{d s^{2}}\left(\varepsilon^{*}, 0\right)+4 a_{\varepsilon} \varphi_{1} \frac{d v}{d s}\left(\varepsilon^{*}, 0\right)-\frac{d^{2} \lambda}{d s^{2}}\left(\varepsilon^{*}, 0\right) \varphi_{1}=0 .
$$

Multiplying this relation by $\varphi_{1}$, integrating in $\Omega$ and applying the formula of integration by parts yields

$$
\frac{d^{2} \lambda}{d s^{2}}\left(\varepsilon^{*}, 0\right)=4 \int_{\Omega} a_{\varepsilon} \varphi_{1}^{2} \frac{d v}{d s}\left(\varepsilon^{*}, 0\right),
$$

since $\int_{\Omega} \varphi_{1}^{2}=1$. On the other hand, specifying $(3.7)$ at $s=0$ and using $\frac{d \lambda}{d s}\left(\varepsilon^{*}, 0\right)=$ 0 , gives

$$
\left(\mathcal{L}-\sigma_{1}^{\Omega}[\mathcal{L}]\right) \frac{d v}{d s}\left(\varepsilon^{*}, 0\right)+a_{\varepsilon}(x) \varphi_{1}^{2}=0 .
$$

By multiplying this relation by $\frac{d v}{d s}\left(\varepsilon^{*}, 0\right)$, integrating in $\Omega$ and applying the formula of integration by parts we obtain

$$
\begin{aligned}
& \sum_{i, j=1}^{N} \int_{\Omega} c_{i j} D_{i} \frac{d v}{d s}\left(\varepsilon^{*}, 0\right) D_{j} \frac{d v}{d s}\left(\varepsilon^{*}, 0\right)+\int_{\Omega}\left(c_{0}-\sigma_{1}^{\Omega}[\mathcal{L}]\right)\left(\frac{d v}{d s}\left(\varepsilon^{*}, 0\right)\right)^{2} \\
& \quad=-\int_{\Omega} a_{\varepsilon} \varphi_{1}^{2} \frac{d v}{d s}\left(\varepsilon^{*}, 0\right) .
\end{aligned}
$$


$\mathrm{u}(\mathrm{s})$

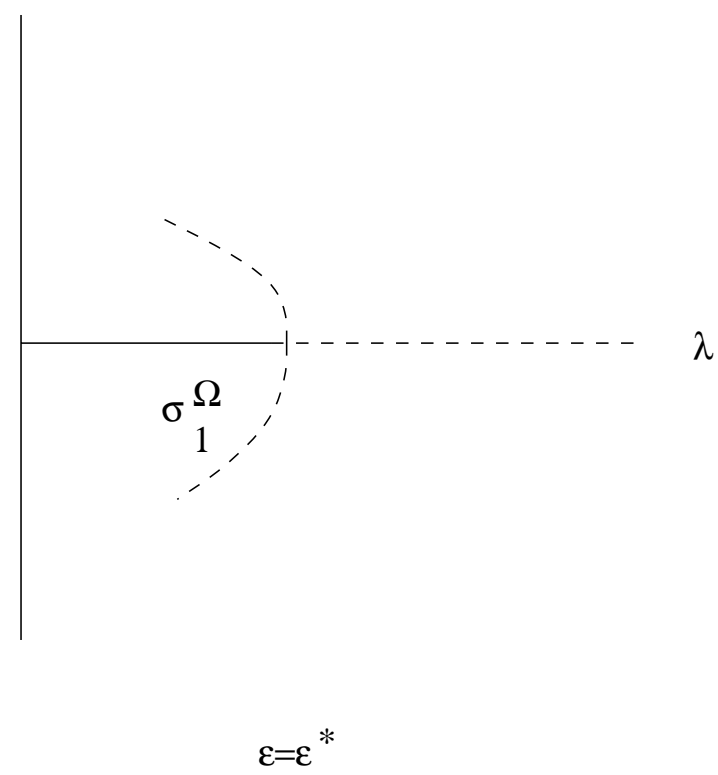

Figure 2. Bifurcation diagram for $\varepsilon=\varepsilon^{*}$.

Moreover, since $a_{\varepsilon} \neq 0,(3.14)$ guarantees that $\frac{d v}{d s}\left(\varepsilon^{*}, 0\right)$ cannot be a multiple of $\varphi_{1}$. Thus, using the variational characterization of $\sigma_{1}^{\Omega}[\mathcal{L}]$ and taking into account that $\varphi_{1}$ is the unique function reaching the infimum, we find that

$$
\sum_{i, j=1}^{N} \int_{\Omega} c_{i j} D_{i} \frac{d v}{d s}\left(\varepsilon^{*}, 0\right) D_{j} \frac{d v}{d s}\left(\varepsilon^{*}, 0\right)+\int_{\Omega} c_{0}\left(\frac{d v}{d s}\left(\varepsilon^{*}, 0\right)\right)^{2}>\sigma_{1}^{\Omega}[\mathcal{L}] \int_{\Omega}\left(\frac{d v}{d s}\left(\varepsilon^{*}, 0\right)\right)^{2},
$$

and hence,

$$
\int_{\Omega} a_{\varepsilon} \varphi_{1}^{2} \frac{d v}{d s}\left(\varepsilon^{*}, 0\right)<0
$$

Thus, (3.13) implies that

$$
\frac{d^{2} \lambda}{d s^{2}}\left(\varepsilon^{*}, 0\right)=4 \int_{\Omega} a_{\varepsilon} \varphi_{1}^{2} \frac{d v}{d s}\left(\varepsilon^{*}, 0\right)<0,
$$

and therefore at $\varepsilon=\varepsilon^{*}$ we have a subcritical pitchfork bifurcation. The corresponding bifurcation diagram is illustrated in Figure 2.

In the figures, continuous lines represent stable solutions and dashed lines unstable solutions. By the exchange stability principle, the two branches of the pitchfork are unstable [9]. If we perturb $\varepsilon$ around $\varepsilon^{*}$ we get an unfolding of the pitchfork as sketched in Figure 3 [15]. For $\varepsilon \neq \varepsilon^{*}$ we know from above that there is a transcritical bifurcation from $u=0$ at $\lambda=\sigma_{1}^{\Omega}[\mathcal{L}]$. Singularity theory provides us with some additional information. The stable branch of bifurcating solutions which exists for $\lambda>\sigma_{1}^{\Omega}[\mathcal{L}]$ quickly turns around and becomes a stable branch. By quickly we mean that the quadratic subcritical turning points converge to $(u, \lambda)=\left(0, \sigma_{1}^{\Omega}[\mathcal{L}]\right)$ 

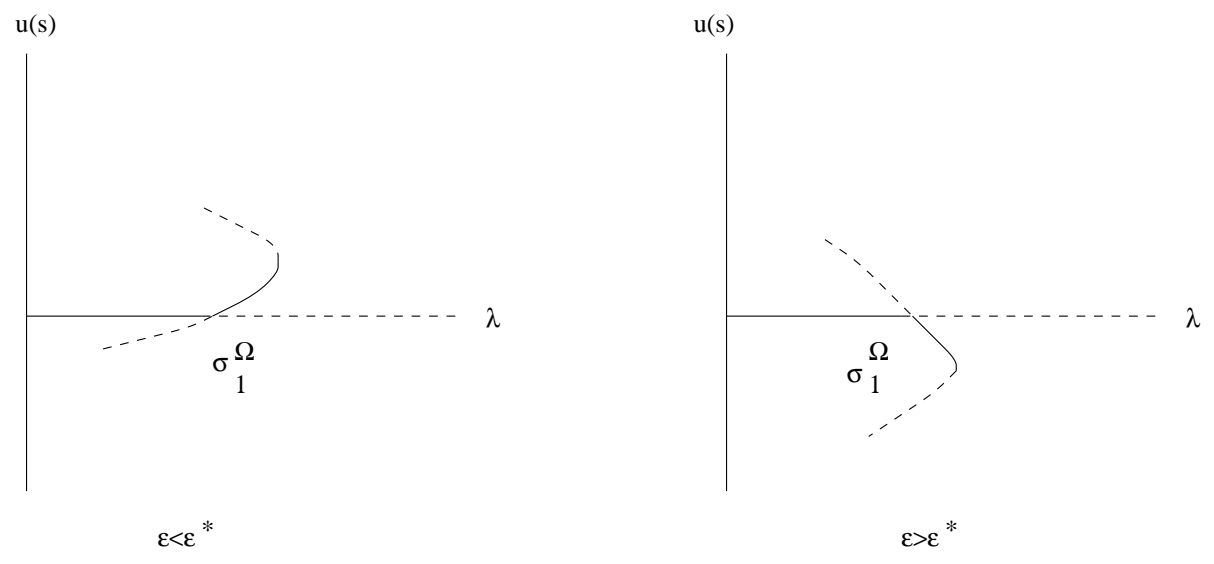

FiguRE 3. Unfolding of the pitchfork.

as $\varepsilon \rightarrow 0$. Note that for $s>0$ the bifurcating solutions are positive, while they are negative for $s<0$.

The previous information can be summarized in the following theorem.

Theorem 3.5. (i) Assume $\varepsilon<\varepsilon^{*}$. Then, there exists $\varepsilon_{1}<\varepsilon^{*}$ such that for any $\varepsilon \in\left(\varepsilon_{1}, \varepsilon^{*}\right)$ one can find a neighborhood $\mathcal{B}$ of $(u, \lambda)=\left(0, \sigma_{1}^{\Omega}[\mathcal{L}]\right)$ in $U \times \mathbb{R}$ and a value $\lambda^{*}>\sigma_{1}^{\Omega}[\mathcal{L}]$ such that

(a) (1.3) does not admit a positive solution in $\mathcal{B}$ if $\lambda>\lambda^{*}$;

(b) (1.3) possesses one positive solution in $\mathcal{B}$ when $\lambda=\lambda^{*}$. Moreover, this solution is neutrally stable;

(c) (1.3) possesses two positive solutions in $\mathcal{B}$ for each $\lambda \in\left(\sigma_{1}^{\Omega}[\mathcal{L}], \lambda^{*}\right)$; the smaller solution is asymptotically stable and the other unstable with one-dimensional unstable manifold;

(d) if $\lambda \leq \sigma_{1}^{\Omega}[\mathcal{L}]$, then (1.3) possesses one positive solution in $\mathcal{B}$ which is unstable with one-dimensional unstable manifold.

(ii) Assume $\varepsilon \geq \varepsilon^{*}$. Then, there exists a neighborhood $\mathcal{B}$ of $(u, \lambda)=\left(0, \sigma_{1}^{\Omega}[\mathcal{L}]\right)$ in $U \times \mathbb{R}$ such that

(a) (1.3) does not admit a positive solution in $\mathcal{B}$ if $\lambda \geq \sigma_{1}^{\Omega}[\mathcal{L}]$;

(b) (1.3) possesses one positive solution in $\mathcal{B}$ for each $\lambda<\sigma_{1}^{\Omega}[\mathcal{L}]$. Moreover, the unstable manifold of this solution is one-dimensional.

In many different contexts experience suggests the local behavior often persists for values of the parameters far away from those for which Theorem 3.5 holds true, which explains why singularity theory has proven so useful. In Sections 4 and 5 we shall give a global version of Theorem 3.5(i). The next generalization of Theorem 2 of 4] shows the global nature of Theorem 3.5(ii)(a).

Theorem 3.6. Assume $\varepsilon \geq \varepsilon^{*}$. Then, (1.3) does not admit a positive solution for $\lambda \geq \sigma_{1}^{\Omega}[\mathcal{L}]$. 
Proof. First, we show that if $u, v \in C_{0}^{1}(\bar{\Omega}) \cap C^{2}(\Omega)$ satisfy $\frac{u}{v} \in C^{1}(\bar{\Omega})$, then for any function $\Psi: \mathbb{R} \rightarrow \mathbb{R}$ of class $C^{1}$ the following identity holds

$$
\int_{\Omega} \Psi\left(\frac{v}{u}\right)[v \mathcal{L} u-u \mathcal{L} v]=-\int_{\Omega} \Psi^{\prime}\left(\frac{v}{u}\right) u^{2} \sum_{i, j=1}^{N} c_{i j} D_{i}\left(\frac{v}{u}\right) D_{j}\left(\frac{v}{u}\right) .
$$

Indeed, rearranging terms gives

$$
\begin{aligned}
\Psi\left(\frac{v}{u}\right)[v \mathcal{L} u-u \mathcal{L} v] & =\Psi\left(\frac{v}{u}\right)\left[u \sum_{i, j=1}^{N} D_{i}\left(c_{i j} D_{j} v\right)-v \sum_{i, j=1}^{N} D_{i}\left(c_{i j} D_{j} u\right)\right] \\
& =\sum_{i=1}^{N} D_{i}\left[\Psi\left(\frac{v}{u}\right) u \sum_{j=1}^{N} c_{i j} D_{j} v-\Psi\left(\frac{v}{u}\right) v \sum_{j=1}^{N} c_{i j} D_{j} u\right] \\
& -\sum_{i=1}^{N}\left[D_{i}\left(\Psi\left(\frac{v}{u}\right) u\right) \sum_{j=1}^{N} c_{i j} D_{j} v-D_{i}\left(\Psi\left(\frac{v}{u}\right) v\right) \sum_{j=1}^{N} c_{i j} D_{j} u\right] .
\end{aligned}
$$

Thus,

$$
\int_{\Omega} \Psi\left(\frac{v}{u}\right)[v \mathcal{L} u-u \mathcal{L} v]=-\sum_{i=1}^{N} \int_{\Omega}\left[D_{i}\left(\Psi\left(\frac{v}{u}\right) u\right) \sum_{j=1}^{N} c_{i j} D_{j} v-D_{i}\left(\Psi\left(\frac{v}{u}\right) v\right) \sum_{j=1}^{N} c_{i j} D_{j} u\right] .
$$

On the other hand, differentiating and rearranging terms gives

$$
\begin{aligned}
\sum_{i=1}^{N} & {\left[D_{i}\left(\Psi\left(\frac{v}{u}\right) u\right) \sum_{j=1}^{N} c_{i j} D_{j} v-D_{i}\left(\Psi\left(\frac{v}{u}\right) v\right) \sum_{j=1}^{N} c_{i j} D_{j} u\right] } \\
= & \sum_{i=1}^{N} \Psi^{\prime}\left(\frac{v}{u}\right) D_{i}\left(\frac{v}{u}\right)\left[u \sum_{j=1}^{N} c_{i j} D_{j} v-v \sum_{j=1}^{N} c_{i j} D_{j} u\right] \\
& +\sum_{i=1}^{N} \Psi\left(\frac{v}{u}\right)\left[D_{i} u \sum_{j=1}^{N} c_{i j} D_{j} v-D_{i} v \sum_{j=1}^{N} c_{i j} D_{j} u\right] \\
= & \Psi^{\prime}\left(\frac{v}{u}\right) u^{2} \sum_{i, j=1}^{N} c_{i j} D_{i}\left(\frac{v}{u}\right) D_{j}\left(\frac{v}{u}\right),
\end{aligned}
$$

since $c_{i j}=c_{j i}$. Substituting this relation into (3.17) we get (3.16).

We now complete the proof of Theorem 3.5. Let $u$ be a positive solution of (1.3). Multiplying (1.3) by $\varphi_{1}$,

$$
\mathcal{L} \varphi_{1}=\sigma_{1}^{\Omega}[\mathcal{L}] \varphi_{1}
$$

by $u$ and substracting the corresponding results yields

$$
\left(\frac{\varphi_{1}}{u}\right)^{2}\left[\varphi_{1} \mathcal{L} u-u \mathcal{L} \varphi_{1}\right]=\left(\frac{\varphi_{1}}{u}\right)^{3} u^{2}\left(\lambda-\sigma_{1}^{\Omega}[\mathcal{L}]\right)-a_{\varepsilon}(x) \varphi_{1}^{3} .
$$

Moreover, it follows from (3.16) that

$$
\int_{\Omega}\left(\frac{\varphi_{1}}{u}\right)^{2}\left[\varphi_{1} \mathcal{L} u-u \mathcal{L} \varphi_{1}\right]=-\int_{\Omega} 2 \frac{\varphi_{1}}{u} u^{2} \sum_{i, j=1}^{N} c_{i j} D_{i}\left(\frac{\varphi_{1}}{u}\right) D_{j}\left(\frac{\varphi_{1}}{u}\right)<0,
$$


since $\mathcal{L}$ is elliptic. In this relation the inequality is strict because $\varphi_{1}$ cannot be a multiple of $u$. On the other hand, $\varepsilon \geq \varepsilon^{*}$ implies

$$
\int_{\Omega} a_{\varepsilon} \varphi_{1}^{3} \leq 0
$$

and therefore we find from (3.18) and (3.19) that

$$
\lambda<\sigma_{1}^{\Omega}[\mathcal{L}] .
$$

This completes the proof.

\section{The structure of the Global arcs of Stable POSITIVE SOlutions}

In this section we fix $\varepsilon>0$ and regard to $\lambda$ as the main continuation parameter to analyze the global structure of the arcs of stable positive solutions of (1.3). It will be shown that these arcs are filled in by linearly asymptotically stable solutions and that some of the following options occur. Either (i) the arc can be globally continued, or (ii) the arc ends in a quadratic turning point, where the positive solution becomes unstable with one-dimensional unstable manifold, or (iii) the arc can be prolongated up to degenerate at $u=0$. If the third option happens, then the degeneration occurs at the value $\lambda=\sigma_{1}^{\Omega}[\mathcal{L}]$ and necessarily $\varepsilon<\varepsilon^{*}$. In particular, these arcs do not have any hysteresis point. All these features are consequences of the next two propositions.

Proposition 4.1. Let $\left(u_{d}, \lambda_{d}\right)$ be a degenerate positive solution of (1.3) such that

$$
\sigma_{1}^{\Omega}\left[\mathcal{L}-\lambda_{d}+2 a_{\varepsilon} u_{d}\right]=0
$$

and let $\psi_{d} \gg 0$ denote the principal eigenfunction associated with this principal eigenvalue. Then, there exist $s_{0}>0$ and an analytic mapping

$$
(u(\cdot), \lambda(\cdot)):\left(-s_{0}, s_{0}\right) \rightarrow U \times \mathbb{R}
$$

such that $(u(0), \lambda(0))=\left(u_{d}, \lambda_{d}\right)$ and $(u(s), \lambda(s))$ is a positive solution of (1.3) for each $s \in\left(-s_{0}, s_{0}\right)$. Moreover, $(u(s), \lambda(s))$ admits a representation of the form

$$
u(s)=u_{d}+s \psi_{d}+\sum_{j=1}^{\infty} s^{j} x_{j}, \quad \lambda(s)=\lambda_{d}+\sum_{j=1}^{\infty} s^{j} \lambda_{j},
$$

where $\lambda_{j} \in \mathbb{R}$ and $x_{j} \in U, j \geq 1$, satisfy

$$
\int_{\Omega} x_{j} \psi_{d}=0, \quad j \geq 1
$$

and there exists a neighborhood $\mathcal{B}$ of $\left(u_{d}, \lambda_{d}\right)$ in $U \times \mathbb{R}$ such that if $(u, \lambda) \in \mathcal{B}$ is a solution of $(1.3)$, then $(u, \lambda)=(u(s), \lambda(s))$ for some $s \in\left(-s_{0}, s_{0}\right)$. Furthermore,

$$
\lambda_{1}=0, \quad x_{1}=0,
$$

and if we normalize $\psi_{d}$ so that $\int_{\Omega} \psi_{d}^{2}=1$, then

$$
\lambda_{2}=\frac{\int_{\Omega} a_{\varepsilon} \psi_{d}^{3}}{\int_{\Omega} u_{d} \psi_{d}}
$$


Proof. The solutions of (1.3) are the zeros of the operator $\mathcal{F}$ defined by

$$
\mathcal{F}(u, \lambda, \varepsilon)=\mathcal{L} u-\lambda u+a_{\varepsilon} u^{2} .
$$

By the simplicity of the principal eigenvalue,

$$
N\left[D_{u} \mathcal{F}\left(u_{d}, \lambda_{d}, \varepsilon\right)\right]=\operatorname{span}\left[\psi_{d}\right] .
$$

We claim that

$$
D_{\lambda} \mathcal{F}\left(u_{d}, \lambda_{d}, \varepsilon\right)=-u_{d} \notin R\left[D_{u} \mathcal{F}\left(u_{d}, \lambda_{d}, \varepsilon\right)\right] .
$$

Indeed, if there exists $u \in U$ satisfying

$$
D_{u} \mathcal{F}\left(u_{d}, \lambda_{d}, \varepsilon\right) u=u_{d},
$$

then multiplying this relation by $\psi_{d}$, integrating in $\Omega$ and applying the formula of integration by parts yields

$$
\int_{\Omega} u_{d} \psi_{d}=0
$$

which is impossible, since the integrand is positive. It can be easily seen that (4.6) implies

$$
N\left[D_{(u, \lambda)} \mathcal{F}\left(u_{d}, \lambda_{d}, \varepsilon\right)\right]=\operatorname{span}\left[\left(\psi_{d}, 0\right)\right] .
$$

We now show that the structure of the solution set around $\left(u_{d}, \lambda_{d}\right)$ possesses the structure of an analytic curve. Let $X$ denote the $L^{2}$-orthogonal of span $\left[\psi_{d}\right]$ in $U$, and set $Z:=X \times \mathbb{R}$. Then, thanks to (4.7) we have

$$
U \times \mathbb{R}=N\left[D_{(u, \lambda)} \mathcal{F}\left(u_{d}, \lambda_{d}, \varepsilon\right)\right] \oplus Z
$$

and hence, each element $(u, \lambda) \in U \times \mathbb{R}$ admits a unique decomposition as

$$
(u, \lambda)=s\left(\psi_{d}, 0\right)+z
$$

where $s \in \mathbb{R}$ and $z \in Z$. Indeed, it suffices to take

$$
z=\left(u-s \psi_{d}, \lambda\right), \quad s=\frac{\int_{\Omega} u \psi_{d}}{\int_{\Omega} \psi_{d}^{2}} .
$$

Thus, the equation $\mathcal{F}(u, \lambda, \varepsilon)=0$ can be written as $\mathcal{H}(z, s)=0$, where

$$
\mathcal{H}(z, s)=\mathcal{F}\left(\left(u_{d}, \lambda_{d}\right)+s\left(\psi_{d}, 0\right)+z\right) .
$$

In this way the solutions $(u, \lambda)$ of $\mathcal{F}=0$ close to $\left(u_{d}, \lambda_{d}\right)$ are in correspondence with the solutions $(z, s)$ of $\mathcal{H}=0$ near $(0,0)$. We have

$$
\mathcal{H}(0,0)=\mathcal{F}\left(u_{d}, \lambda_{d}\right)=0 .
$$

Moreover, the operator

$$
D_{z} \mathcal{H}(0,0)=\left.D_{(u, \lambda)} \mathcal{F}\left(u_{d}, \lambda_{d}, \varepsilon\right)\right|_{Z}
$$

is a topological isomorphism. Thus, by the implicit function theorem, there exist $s_{0}>0$ and an analytic mapping $z:\left(-s_{0}, s_{0}\right) \rightarrow Z$ such that $z(0)=0$ and $\mathcal{H}(z(s), s)=0$ for each $s \in\left(-s_{0}, s_{0}\right)$. Moreover, there is a neighborhood $B$ of $(0,0)$ in $Z \times \mathbb{R}$ such that if $(z, s) \in B$ satisfies $\mathcal{H}(z, s)=0$, then $z=z(s)$. Therefore, in a neighborhood $\mathcal{B}$ of $\left(u_{d}, \lambda_{d}\right)$ the solution set $\mathcal{F}(\cdot, \cdot, \varepsilon)=0$ is constituted by an analytic curve $(u(s), \lambda(s))$ which admits a parametrization of the form (4.2) with $x_{j} \in X$ and $\lambda_{j} \in \mathbb{R}$ for all $j \geq 1$. Condition (4.3) follows from the definition of $X$. 
To complete the proof it suffices to show (4.4) and (4.5). Substituting (4.2) into (1.3), we find that

$$
\begin{aligned}
\mathcal{L}\left(u_{d}\right. & \left.+s\left(\psi_{d}+x_{1}\right)+\sum_{j=2}^{\infty} s^{j} x_{j}\right) \\
= & {\left[\lambda_{d}+\sum_{j=1}^{\infty} s^{j} \lambda_{j}-a_{\varepsilon}\left(u_{d}+s\left(\psi_{d}+x_{1}\right)+\sum_{j=2}^{\infty} s^{j} x_{j}\right)\right] } \\
\cdot & {\left.\left[u_{d}+s\left(\psi_{d}+x_{1}\right)+\sum_{j=2}^{\infty} s^{j} x_{j}\right)\right] }
\end{aligned}
$$

for all $s \simeq 0$. Thus, identifying terms up to the second order yields

$$
\begin{gathered}
\mathcal{L} u_{d}=\left(\lambda_{d}-a_{\varepsilon} u_{d}\right) u_{d}, \\
\mathcal{L}\left(\psi_{d}+x_{1}\right)=\left(\lambda_{d}-a_{\varepsilon} u_{d}\right)\left(\psi_{d}+x_{1}\right)+\left(\lambda_{1}-a_{\varepsilon}\left(\psi_{d}+x_{1}\right)\right) u_{d}, \\
\mathcal{L} x_{2}=\left(\lambda_{d}-a_{\varepsilon} u_{d}\right) x_{2}+\left(\lambda_{1}-a_{\varepsilon}\left(\psi_{d}+x_{1}\right)\right)\left(\psi_{d}+x_{1}\right)+\left(\lambda_{2}-a_{\varepsilon} x_{2}\right) u_{d} .
\end{gathered}
$$

Condition (4.9a) is the same as $\mathcal{F}\left(u_{d}, \lambda_{d}, \varepsilon\right)=0$ and so it does not give any additional information. By rearranging $(4.9 \mathrm{~b})$ we find that

$$
\left(\mathcal{L}+2 a_{\varepsilon} u_{d}-\lambda_{d}\right)\left(\psi_{d}+x_{1}\right)=\lambda_{1} u_{d} .
$$

Thanks to (4.6), it follows from (4.10) that

$$
\lambda_{1}=0
$$

and so $\psi_{d}+x_{1} \in N\left[D_{u} \mathcal{F}\left(u_{d}, \lambda_{d}\right)\right]$. Hence, there exists $c \in \mathbb{R}$ such that

$$
\psi_{d}+x_{1}=c \psi_{d} .
$$

Multiplying this relation by $\psi_{d}$ and integrating in $\Omega$ we find from (4.3) that $c=1$ and therefore,

$$
x_{1}=0 .
$$

This completes the proof of (4.4). Now, substituting (4.4) into (4.9c) and rearranging terms gives

$$
\left(\mathcal{L}+2 a_{\varepsilon} u_{d}-\lambda_{d}\right) x_{2}=\lambda_{2} u_{d}-a_{\varepsilon} \psi_{d}^{2} .
$$

Finally, multiplying this relation by $\psi_{d}$, integrating in $\Omega$ and applying the formula of integration by parts, we get (4.5). This completes the proof.

If we assume $\lambda_{2}=0$ and $\lambda_{3} \neq 0$, for instance $\lambda_{3}<0$, then the curve of solutions passing through by $\left(u_{d}, \lambda_{d}\right)$ appears as illustrated in Figure 4, where a genuine cubic hysteresis point is shown.

Note that $\frac{d u}{d s}(s)=\psi_{d}+O(s) \gg 0$ and that $\lambda(s)=\lambda_{d}+\lambda_{3} s^{3}+O\left(s^{4}\right)$. So, $\lambda(s)>\lambda_{d}$ if $s<0$, and $\lambda(s)<\lambda_{d}$ if $s>0$. If we now vary a further parameter, for instance $\varepsilon$, then the diagram of Figure 4 will generically degenerate into a curve of some of the two types illustrated in Figure 5.

The next result shows that these situations cannot occur if some of the half-curves of solutions passing through by $\left(u_{d}, \lambda_{d}\right)$ are filled in by linearly asymptotically stable 


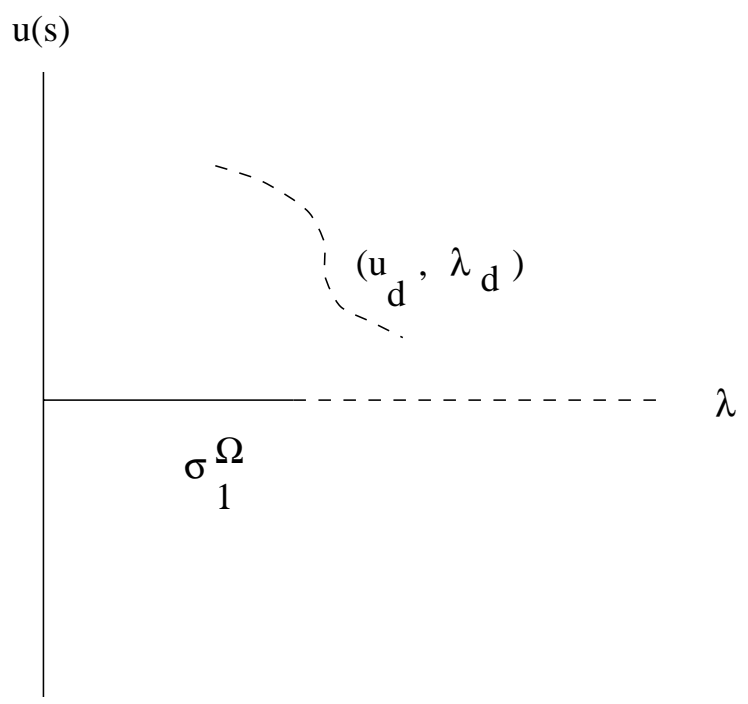

Figure 4. An hysteresis point.
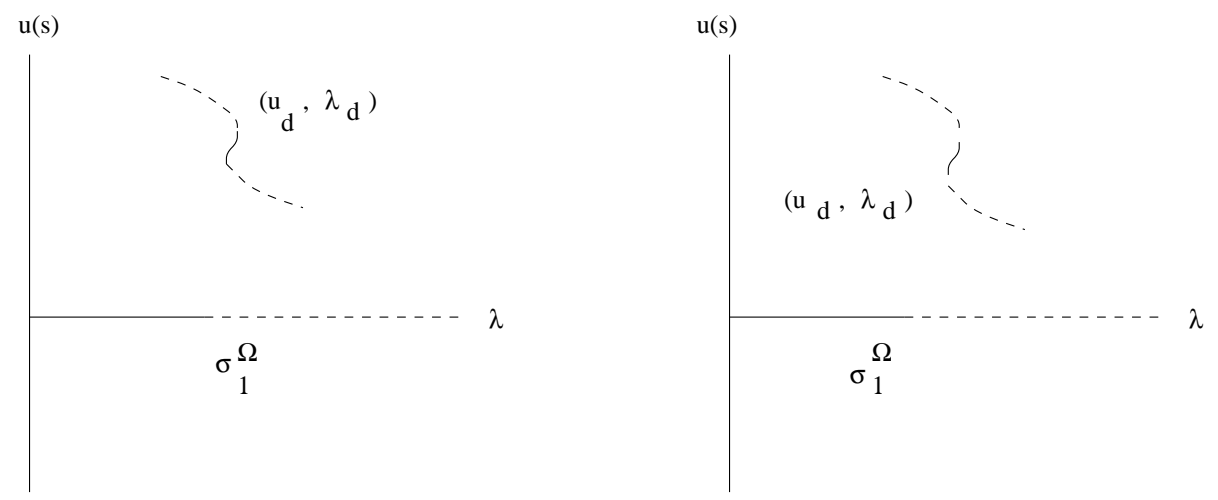

Figure 5. The unfolding of a cubic.

solutions. Notice that since the mapping $s \rightarrow \sigma_{1}^{\Omega}\left[D_{u} \mathcal{F}(u(s), \lambda(s), \varepsilon)\right]$ is analytic, some of the following options occur. Either

$$
\sigma_{1}^{\Omega}\left[D_{u} \mathcal{F}(u(s), \lambda(s), \varepsilon)\right] \cdot \sigma_{1}^{\Omega}\left[D_{u} \mathcal{F}(u(-s), \lambda(-s), \varepsilon)\right]>0,
$$

for all $s$ sufficiently small, or

$$
\sigma_{1}^{\Omega}\left[D_{u} \mathcal{F}(u(s), \lambda(s), \varepsilon)\right] \cdot \sigma_{1}^{\Omega}\left[D_{u} \mathcal{F}(u(-s), \lambda(-s), \varepsilon)\right]<0,
$$

for all $s$ sufficiently small, or

$$
\sigma_{1}^{\Omega}\left[D_{u} \mathcal{F}(u(s), \lambda(s), \varepsilon)\right]=0,
$$

for all $s$ sufficiently small. Condition (4.12) means that the two half-curves are filled in by either linearly asymptotically stable states or by linearly unstable states. Condition (4.13) means that some of the half-curves are filled in by linearly asymptotically stable solutions while the other is formed by linearly unstable solutions. 
Condition (4.14) means that the two half-curves are filled in by neutrally stable states, and it arises when we have a vertical diagram of solutions. The latest case cannot occur in our current situation, because in this case by Proposition 4.1 we could continue the curve of positive solutions up to reach $u=0$ and therefore $\lambda_{d}=\sigma_{1}^{\Omega}[\mathcal{L}]$, which is impossible because thanks to the local analysis of Section 2 we already know that no vertical bifurcation occurs from $u=0$. The next result shows that if some of the half-curves are formed by linearly asymptotically stable states, then $\left(u_{d}, \lambda_{d}\right)$ is a quadratic turning point, i.e., $\lambda_{2} \neq 0$, and vice versa. Therefore, hysteresis points are only possible on arcs of solution curves filled in by linearly unstable solutions.

Proposition 4.2. Under the assumptions of Proposition 4.1, the following assertions are true:

(i) $\lambda_{2}<0$ if, and only if,

$$
\sigma_{1}^{\Omega}\left[D_{u} \mathcal{F}(u(s), \lambda(s), \varepsilon)\right]>0 \quad \text { for } \quad s<0 .
$$

Moreover, in this case we have

$$
\sigma_{1}^{\Omega}\left[D_{u} \mathcal{F}(u(s), \lambda(s), \varepsilon)\right]<0 \quad \text { for } \quad s>0 .
$$

(ii) $\lambda_{2}>0$ if, and only if,

$$
\sigma_{1}^{\Omega}\left[D_{u} \mathcal{F}(u(s), \lambda(s), \varepsilon)\right]>0 \quad \text { for } \quad s>0 .
$$

Moreover, in this case we have

$$
\sigma_{1}^{\Omega}\left[D_{u} \mathcal{F}(u(s), \lambda(s), \varepsilon)\right]<0 \quad \text { for } \quad s<0 .
$$

In particular, $\left(u_{d}, \lambda_{d}\right)$ is a quadratic turning point if and only if, some of the halfcurves are filled in by linearly asymptotically stable solutions. Moreover, if this is the case, then the other half-curve is formed by linearly unstable solutions.

Proof. First, we show that $\lambda_{2}=0$ implies

$$
\lambda_{3}<0 .
$$

Suppose $\lambda_{2}=0$. Then, (4.11) reduces to

$$
\left(\mathcal{L}+2 a_{\varepsilon} u_{d}-\lambda_{d}\right) x_{2}=-a_{\varepsilon} \psi_{d}^{2}
$$

Multiplying this relation by $x_{2}$, integrating in $\Omega$ and applying the formula of integration by parts, gives

$$
\sum_{i, j=1}^{N} \int_{\Omega} c_{i j} D_{i} x_{2} D_{j} x_{2}+\int_{\Omega}\left(c_{0}+2 a_{\varepsilon} u_{d}-\lambda_{d}\right) x_{2}^{2}=-\int_{\Omega} a_{\varepsilon} \psi_{d}^{2} x_{2} .
$$

By (4.18), $x_{2} \neq 0$. Moreover, since $\int_{\Omega} \psi_{d} x_{2}=0, x_{2}$ cannot be a multiple of $\psi_{d}$. On the other hand, by the variational characterization of $\sigma_{1}^{\Omega}\left[\mathcal{L}+2 a_{\varepsilon} u_{d}-\lambda_{d}\right]$,

$$
\begin{aligned}
\sigma_{1}^{\Omega}[\mathcal{L} & \left.+2 a_{\varepsilon} u_{d}-\lambda_{d}\right] \\
= & \inf _{\psi \in H_{0}^{1}(\Omega)}\left\{\sum_{i, j=1}^{N} \int_{\Omega} c_{i j} D_{i} \psi D_{j} \psi+\int_{\Omega}\left(c_{0}+2 a_{\varepsilon} u_{d}-\lambda_{d}\right) \psi^{2}, \int_{\Omega} \psi^{2}=1\right\},
\end{aligned}
$$

being the infimum if and only if $\psi=\psi_{d}$. Hence, due to (4.1) we get

$$
\sum_{i, j=1}^{N} \int_{\Omega} c_{i j} D_{i} x_{2} D_{j} x_{2}+\int_{\Omega}\left(c_{0}+2 a_{\varepsilon} u_{d}-\lambda_{d}\right) x_{2}^{2}>\sigma_{1}^{\Omega}\left[\mathcal{L}+2 a_{\varepsilon} u_{d}-\lambda_{d}\right] \int_{\Omega} x_{2}^{2}=0
$$


and so the right-hand side of (4.19) is positive. Therefore,

$$
\int_{\Omega} a_{\varepsilon} \psi_{d}^{2} x_{2}<0
$$

Now, going back to (4.8) and identifying third order terms yields

$$
\begin{aligned}
\mathcal{L} x_{3}= & \left(\lambda_{d}-a_{\varepsilon} u_{d}\right) x_{3}+\left(\lambda_{1}-a_{\varepsilon}\left(\psi_{d}+x_{1}\right)\right) x_{2} \\
& +\left(\lambda_{2}-a_{\varepsilon} x_{2}\right)\left(\psi_{d}+x_{1}\right)+\left(\lambda_{3}-a_{\varepsilon} x_{3}\right) u_{d} .
\end{aligned}
$$

Since $x_{1}=0$ and $\lambda_{1}=\lambda_{2}=0$, this relation reduces to

$$
\left(\mathcal{L}+2 a_{\varepsilon} u_{d}-\lambda_{d}\right) x_{3}=-2 a_{\varepsilon} \psi_{d} x_{2}+\lambda_{3} u_{d}
$$

and hence

$$
\lambda_{3} \int_{\Omega} u_{d} \psi_{d}=2 \int_{\Omega} a_{\varepsilon} \psi_{d}^{2} x_{2} .
$$

Thanks to (4.20) the right-hand side of this relation is negative and therefore (4.17) is proven.

Now, by differentiating $\mathcal{F}(u(s), \lambda(s), \varepsilon)=0$ with respect to $s$ we find that

$$
\left(\mathcal{L}-\lambda(s)+2 a_{\varepsilon} u(s)\right) \frac{d u}{d s}(s)=\frac{d \lambda}{d s}(s) u(s) .
$$

Moreover, from (4.2) we have

$$
\frac{d u}{d s}(s)=\psi_{d}+O(s) \gg 0, \quad \frac{d \lambda}{d s}(s)=2 s \lambda_{2}+3 s^{2} \lambda_{3}+O\left(s^{3}\right) .
$$

Assume equation (4.15) and pick up $s<0$. Since $u(s) \gg 0, \frac{d u}{d s}(s) \gg 0$ and $\sigma_{1}^{\Omega}\left[\mathcal{L}-\lambda(s)+2 a_{\varepsilon} u(s)\right]>0$, applying Theorem 2.2 to (4.21) gives

$$
\frac{d \lambda}{d s}(s)>0 \text {. }
$$

Thus, it follows from (4.22) that $\lambda_{2} \leq 0$. Moreover, if $\lambda_{2}=0$, then $\lambda_{3}<0$ and this contradicts (4.23). Therefore, $\lambda_{2}<0$. The same argument shows that $\lambda_{2}>0$ if (4.16) is satisfied.

We now show the contrary. Assume $\lambda_{2}<0$. Then, it follows from (4.22) that $\frac{d \lambda}{d s}(s)>0$ if $s<0$ and that $\frac{d \lambda}{d s}(s)<0$ if $s>0$. Thus, we find from (4.21) that

$$
\left(\mathcal{L}-\lambda(s)+2 a_{\varepsilon} u(s)\right) \frac{d u}{d s}(s) \gg 0 \quad \text { if } s<0,
$$

and that

$$
\left(\mathcal{L}-\lambda(s)+2 a_{\varepsilon} u(s)\right) \frac{d u}{d s}(s) \ll 0 \quad \text { if } s>0 .
$$

Therefore, thanks to Theorem 2.2,

$$
\sigma_{1}^{\Omega}\left[\mathcal{L}-\lambda(s)+2 a_{\varepsilon} u(s)\right]>0 \quad \text { if } s<0,
$$

and

$$
\sigma_{1}^{\Omega}\left[\mathcal{L}-\lambda(s)+2 a_{\varepsilon} u(s)\right]<0 \quad \text { if } s>0 .
$$

This argument can be easily adapted to complete the proof of part (ii). 
We now show how the previous propositions provide us with the structure of the arcs of the curve filled in by stable solutions. Fix $\varepsilon>0$ and assume that $\left(u_{0}, \lambda_{0}\right)$ is a positive solution of (1.3) linearly asymptotically stable, i.e.,

$$
\sigma_{1}^{\Omega}\left[\mathcal{L}-\lambda_{0}+2 a_{\varepsilon}(x) u_{0}(x)\right]>0 .
$$

We have $\mathcal{F}\left(u_{0}, \lambda_{0}, \varepsilon\right)=0$ and thanks to (4.26) the operator

$$
D_{u} \mathcal{F}\left(u_{0}, \lambda_{0}, \varepsilon\right)=\mathcal{L}-\lambda_{0}+2 a_{\varepsilon}(x) u_{0}(x)
$$

is an isomorphism. Thus, through by $\left(u_{0}, \lambda_{0}\right)$ passes a real analytic curve of positive solutions of (1.3) of the form $u=u(\lambda), \lambda \simeq \lambda_{0}$. Moreover, the solutions on this curve provide us with all the solutions of (1.3) in a neighborhood of $\left(u_{0}, \lambda_{0}\right)$. Differentiating $\mathcal{F}(u(\lambda), \lambda, \varepsilon)=0$ with respect to $\lambda$ at $\lambda_{0}$ gives

$$
\left(\mathcal{L}-\lambda_{0}+2 a_{\varepsilon}(x) u_{0}(x)\right) \frac{d u}{d \lambda}\left(\lambda_{0}\right)=u_{0} \gg 0
$$

and hence, the strong maximum principle implies

$$
\frac{d u}{d \lambda}\left(\lambda_{0}\right)=\left(\mathcal{L}-\lambda_{0}+2 a_{\varepsilon}(x) u_{0}(x)\right)^{-1}\left(u_{0}\right) \gg 0 .
$$

Therefore, the mapping $\lambda \rightarrow u(\lambda)$ is increasing in $C_{0}(\bar{\Omega})$. This analysis shows that as soon as the positive solution is linearly asymptotically stable we can continue the curve of positive solutions for larger values of $\lambda$. We already know that this curve cannot be prolongated beyond the critical value $\sigma_{1}^{\Omega \backslash \Omega_{+}}[\mathcal{L}]$. Therefore, the supremum of the set of $\lambda$ for which the curve can be prolongated is well defined. Let $\lambda_{\omega}$ denote it. We have $\lambda_{\omega} \in\left(\lambda_{0}, \sigma_{1}^{\Omega \backslash \Omega_{+}}[\mathcal{L}]\right]$. Moreover, some of the following options occur. Either

$$
\lim _{\lambda \uparrow \lambda_{\omega}}\|u(\lambda)\|_{\infty}=\infty
$$

Or

$$
\sigma_{1}^{\Omega}\left[\mathcal{L}-\lambda+2 a_{\varepsilon} u(\lambda)\right]>0 \quad \text { for all } \lambda \in\left[\lambda_{0}, \lambda_{\omega}\right)
$$

and

$$
\sigma_{1}^{\Omega}\left[\mathcal{L}-\lambda_{\omega}+2 a_{\varepsilon} u\left(\lambda_{\omega}\right)\right]=0 .
$$

Note that since (1.3) can be regarded as a fixed point equation for a compact operator if $u(\lambda)$ is uniformly bounded in $\lambda$, then the solutions converge to another solution. Moreover, the limiting solution is positive because $u(\lambda)>u_{0} \gg 0$ for $\lambda>\lambda_{0}$ and since (1.3) does not admit a positive solution for $\lambda=\sigma_{1}^{\Omega \backslash \Omega_{+}}[\mathcal{L}]$, $\lambda_{\omega}<\sigma_{1}^{\Omega \backslash \Omega_{+}}[\mathcal{L}]$. Thanks to Theorem 2.6, this estimate of $\lambda_{\omega}$ is also true when (4.27) occurs and $\varepsilon>0$. Propositions 4.1 and 4.2 provide us with the following result.

Theorem 4.3. Suppose (4.28) and (4.29). Then, $\left(u\left(\lambda_{\omega}\right), \lambda_{\omega}\right)$ is a subcritical quadratic turning point of the curve of positive solutions passing through by it. In other words, there exists a neighborhood $\mathcal{B}_{\omega}$ of $\left(u\left(\lambda_{\omega}\right), \lambda_{\omega}\right)$ in $U \times \mathbb{R}$ such that the following assertions are true:

(i) (1.3) does not admit a positive solution in $\mathcal{B}_{\omega}$ if $\lambda>\lambda_{\omega}$;

(ii) (1.3) possesses one positive solution in $\mathcal{B}_{\omega}$ if $\lambda=\lambda_{\omega}$. Moreover, this solution is neutrally stable;

(iii) for each $\lambda<\lambda_{\omega}$, problem (1.3) possesses two positive solutions in $\mathcal{B}_{\omega}$, say $u_{1}$ and $u_{2}$. Moreover, they are ordered and, if we assume that $u_{1} \ll u_{2}$, then $u_{1}=u(\lambda)$ and $u_{2}$ is unstable with one-dimensional unstable manifold. 


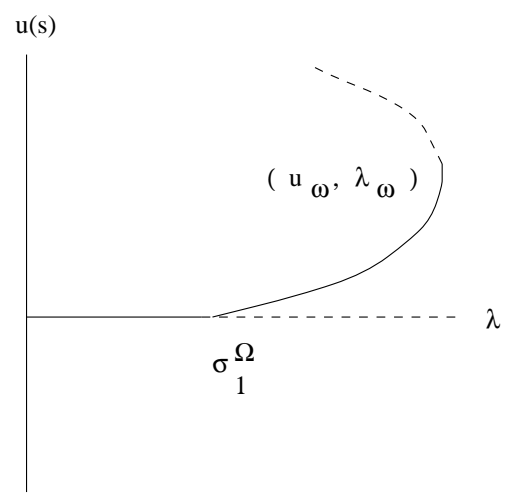

(a)

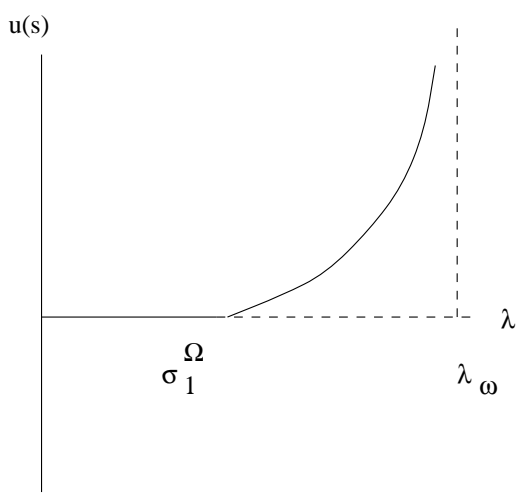

(b)

Figure 6. Possible curves of positive solutions.

Now, let $\lambda_{\alpha}$ denote the infimum of the set of $\lambda<\lambda_{0}$ for which $u(\lambda)$ is well defined and

$$
\sigma_{1}^{\Omega}\left[\mathcal{L}-\lambda+2 a_{\varepsilon} u(\lambda)\right]>0 .
$$

Either $\lambda_{\alpha}=-\infty$, or $\lambda_{\alpha} \in \mathbb{R}$. In general, the first option is not excluded [14]. Assume $\lambda_{\alpha} \in \mathbb{R}$. Then, thanks to the implicit function theorem

$$
\sigma_{1}^{\Omega}\left[\mathcal{L}-\lambda_{\alpha}+2 a_{\varepsilon} u\left(\lambda_{\alpha}\right)\right]=0 .
$$

Since the mapping $\lambda \rightarrow u(\lambda)$ is increasing, $u\left(\lambda_{\alpha}\right)$ is well defined and some of the following options occurs. Either $u\left(\lambda_{\alpha}\right)=0$, or $u\left(\lambda_{\alpha}\right) \gg 0$. Moreover, when $u_{\alpha}=0$ necessarily $\lambda_{\alpha}=\sigma_{1}^{\Omega}[\mathcal{L}]$ and $\varepsilon<\varepsilon^{*}$. If $u\left(\lambda_{\alpha}\right) \gg 0$, then Propositions 4.1 and 4.2 provide us with the following result.

Theorem 4.4. Suppose $\lambda_{\alpha} \in \mathbb{R}$ and $u\left(\lambda_{\alpha}\right) \gg 0$. Then, $\left(u\left(\lambda_{\alpha}\right), \lambda_{\alpha}\right)$ is a supercritical quadratic turning point of the curve of positive solutions passing through it. In other words, there exists a neighborhood $\mathcal{B}_{\alpha}$ of $\left(u\left(\lambda_{\alpha}\right), \lambda_{\alpha}\right)$ in $U \times \mathbb{R}$ such that the following assertions are true:

(i) For $\lambda<\lambda_{\alpha}$ (1.3) does not admit a positive solution in $\mathcal{B}_{\alpha}$.

(ii) (1.3) possesses one positive solution in $\mathcal{B}_{\alpha}$ if $\lambda=\lambda_{\omega}$. Moreover, this solution is neutrally stable.

(iii) For each $\lambda>\lambda_{\alpha}$, problem (1.3) possesses two positive solutions in $\mathcal{B}_{\alpha}$, say $u_{1}$ and $u_{2}$. Moreover, they are ordered and, if we assume that $u_{1} \ll u_{2}$, then $u_{2}=u(\lambda)$ and $u_{1}$ is unstable with one-dimensional unstable manifold.

\section{Global Continuation in $\varepsilon$ OF the Bifurcation diagrams OF POSITIVE SOLUTIONS}

By the analysis done in Section 4 we already know that for $\varepsilon<\varepsilon^{*}$ the curve of positive solutions bifurcating from zero is of one of the two types shown in Figure 6.

Moreover, thanks to Theorem 3.5 we know that if $\varepsilon^{*}-\varepsilon>0$ is sufficiently small, then the curve is as shown in Figure 6(a). In this section we refer to $\varepsilon$ as the main continuation parameter to analyze how varies the curve shown in Figure 6(a), as $\varepsilon$ 
decreases from $\varepsilon^{*}$ to 0 . Our main theorem says that this diagram persists until $\varepsilon$ reaches a critical value $\varepsilon_{*} \in\left[0, \varepsilon^{*}\right)$ at which

$$
\lim _{\varepsilon \downarrow \varepsilon_{*}}\|u[\varepsilon]\|_{\infty}=\infty
$$

where $(u[\varepsilon], \lambda[\varepsilon])$ is the first degenerate solution on the curve of positive solutions bifurcating from $u=0$. Thanks to Propositions 4.1 and $4.2,(u[\varepsilon], \lambda[\varepsilon])$ is a subcritical turning point where the solution becomes unstable. The next result shows the local persistence of the bifurcation diagram and it will provide us with the key to get the main result. The solutions of (1.3) will be denoted by $(u, \lambda, \varepsilon)$.

Theorem 5.1. Fix $\varepsilon_{0} \in\left(0, \varepsilon^{*}\right)$ and let $\left(u(\lambda), \lambda, \varepsilon_{0}\right)$ be a curve of positive solutions of (1.3) defined for $\lambda \in\left(\sigma_{1}^{\Omega}[\mathcal{L}], \lambda_{0}\right]$ satisfying

$$
\begin{gathered}
u\left(\sigma_{1}^{\Omega}[\mathcal{L}]\right)=0, \\
\sigma_{1}^{\Omega}\left[D_{u} \mathcal{F}\left(u(\lambda), \lambda, \varepsilon_{0}\right)\right]>0, \quad \text { for } \quad \lambda \in\left(\sigma_{1}^{\Omega}[\mathcal{L}], \lambda_{0}\right),
\end{gathered}
$$

and

$$
\sigma_{1}^{\Omega}\left[D_{u} \mathcal{F}\left(u_{0}, \lambda_{0}, \varepsilon_{0}\right)\right]=0,
$$

where $u_{0}:=u\left(\lambda_{0}\right)$. Then, there exist $\delta>0$ and a unique analytic mapping

$$
(u[\cdot], \lambda[\cdot]):\left(\varepsilon_{0}-\delta, \varepsilon_{0}+\delta\right) \rightarrow U \times \mathbb{R}
$$

satisfying the following conditions:

(i) For each $\varepsilon \in\left(\varepsilon_{0}-\delta, \varepsilon_{0}+\delta\right),(u[\varepsilon], \lambda[\varepsilon], \varepsilon)$ is a positive solution of (1.3) such that

$$
\sigma_{1}^{\Omega}\left[D_{u} \mathcal{F}(u[\varepsilon], \lambda[\varepsilon], \varepsilon)\right]=0 ;
$$

(ii) $\left(u\left[\varepsilon_{0}\right], \lambda\left[\varepsilon_{0}\right]\right)=\left(u_{0}, \lambda_{0}\right)$;

(iii) $(u[\varepsilon], \lambda[\varepsilon])$ is the first degenerate solution on the curve of positive solutions bifurcating from $u=0$ at $\lambda=\sigma_{1}^{\Omega}[\mathcal{L}]$;

(iv) the mapping $\varepsilon \rightarrow \lambda[\varepsilon]$ is decreasing.

Remark 5.2. (a) Thanks to Theorem 3.5 , for $\varepsilon<\varepsilon^{*}$ sufficiently close, we are under the assumptions of Theorem 5.1.

(b) Condition (iii) means that if $(u(\lambda), \lambda, \varepsilon)$ is the curve of positive solutions bifurcating from $u=0$, then it is defined for all $\lambda \in\left(\sigma_{1}^{\Omega}[\mathcal{L}], \lambda[\varepsilon]\right], u(\lambda[\varepsilon])=u[\varepsilon]$, $\sigma_{1}^{\Omega}\left[D_{u} \mathcal{F}(u(\lambda), \lambda, \varepsilon)\right]>0$ for $\lambda<\lambda[\varepsilon]$, and $\sigma_{1}^{\Omega}\left[D_{u} \mathcal{F}(u[\varepsilon], \lambda[\varepsilon], \varepsilon)\right]=0$.

(c) Thanks to Propositions 4.1 and 4.2, for each $\varepsilon \in\left(\varepsilon_{0}-\delta, \varepsilon_{0}+\delta\right)$ the couple $(u[\varepsilon], \lambda[\varepsilon])$ is a subcritical quadratic turning point of the corresponding curve of positive solutions bifurcating from $u=0$.

Proof of Theorem 5.1. Let $\psi_{0} \gg 0$ denote the principal eigenfunction associated with $\sigma_{1}^{\Omega}\left[D_{u} \mathcal{F}\left(u_{0}, \lambda_{0}, \varepsilon_{0}\right)\right]$, normalized so that $\int_{\Omega} \psi_{0}^{2}=1$. Then,

$$
N\left[D_{u} \mathcal{F}\left(u_{0}, \lambda_{0}, \varepsilon_{0}\right)\right]=\operatorname{span}\left[\psi_{0}\right] .
$$

Consider the operator $\mathcal{F}_{T}: U^{2} \times \mathbb{R}^{2} \rightarrow V^{2} \times \mathbb{R}$ defined by

$$
\mathcal{F}_{T}(u, \psi, \lambda, \varepsilon)=\left(\begin{array}{c}
\mathcal{F}(u, \lambda, \varepsilon) \\
D_{u} \mathcal{F}(u, \lambda, \varepsilon) \psi \\
\int_{\Omega} \psi \psi_{0}-1
\end{array}\right) .
$$


$\mathcal{F}_{T}$ is an analytic operator whose zeros are in correspondence with the degenerate solutions of (1.3). By construction,

$$
\mathcal{F}_{T}\left(u_{0}, \psi_{0}, \lambda_{0}, \varepsilon_{0}\right)=0 .
$$

Moreover,

$$
D_{(u, \psi, \lambda)} \mathcal{F}_{T}\left(u_{0}, \psi_{0}, \lambda_{0}, \varepsilon_{0}\right)=\left(\begin{array}{ccc}
D_{u} \mathcal{F} & 0 & D_{\lambda} \mathcal{F} \\
D_{u u} \mathcal{F}\left\langle\psi_{0}, \cdot\right\rangle & D_{u} \mathcal{F} & D_{u \lambda} \mathcal{F} \psi_{0} \\
0 & \int_{\Omega} \psi_{0} \cdot d x & 0
\end{array}\right),
$$

where all the entries of this matrix are evaluated at $\left(u_{0}, \psi_{0}, \lambda_{0}, \varepsilon_{0}\right)$. In the sequel to short notations we will not make this dependence explicit. We now show that (5.4) is a topological isomorphism. Since (5.4) is linear and continuous, thanks to the open mapping theorem, it suffices to show that (5.4) is a bijection. Given

$$
(\hat{u}, \hat{\psi}, \hat{\lambda}) \in V^{2} \times \mathbb{R}
$$

we have to see that there exists a unique $(u, \psi, \lambda) \in U^{2} \times \mathbb{R}$ satisfying the following

$$
D_{u} \mathcal{F} u+\lambda D_{\lambda} \mathcal{F}=\hat{u},
$$

$$
D_{u u} \mathcal{F}\left\langle\psi_{0}, u\right\rangle+D_{u} \mathcal{F} \psi+\lambda D_{u \lambda} \mathcal{F} \psi_{0}=\hat{\psi},
$$

$$
\int_{\Omega} \psi_{0} \psi d x=\hat{\lambda}
$$

By the definition of $\mathcal{F},(5.5 \mathrm{a})$ and $(5.5 \mathrm{~b})$ can be written in the form

$$
\begin{gathered}
\left(\mathcal{L}-\lambda_{0}+2 a_{\varepsilon_{0}} u_{0}\right) u-\lambda u_{0}=\hat{u}, \\
2 a_{\varepsilon_{0}} \psi_{0} u+\left(\mathcal{L}-\lambda_{0}+2 a_{\varepsilon_{0}} u_{0}\right) \psi-\lambda \psi_{0}=\hat{\psi} .
\end{gathered}
$$

Equation (5.6a) admits a solution $u \in U$ if, and only if,

$$
\lambda=-\frac{\int_{\Omega} \hat{u} \psi_{0}}{\int_{\Omega} u_{0} \psi_{0}} .
$$

Moreover, if we give this value to $\lambda$, then there exists a unique solution of (5.6a) in the $L^{2}$-orthogonal of $\psi_{0}$ in $U$, say $u_{\text {orth }}$, and in fact any solution of (5.6) admits a unique decomposition as

$$
u=\alpha \psi_{0}+u_{\text {orth }}
$$

where $\alpha \in \mathbb{R}$. So, give to $\lambda$ the value (5.7). Let $X$ denote the $L^{2}$-orthogonal of $\psi_{0}$ in $U$. In fact, any $\psi \in U$ possesses a unique decomposition as

$$
\psi=\beta \psi_{0}+\psi_{\text {orth }},
$$

where $\beta \in \mathbb{R}$ and $\psi_{\text {orth }} \in X$. Substituting (5.9) into (5.5c) and using $\int_{\Omega} \psi_{0}^{2}=1$, we find that $\beta=\hat{\lambda}$. Therefore, the set of solutions of (5.5c) is given by

$$
\psi=\hat{\lambda} \psi_{0}+\psi_{\text {orth }}, \quad \psi_{\text {orth }} \in X .
$$

Now, substituting (5.8) and (5.10) into (5.6b) we are reduced to the problem of finding out $\psi_{\text {orth }} \in X$ and $\alpha \in \mathbb{R}$ satisfying the following:

$$
\left(\mathcal{L}-\lambda_{0}+2 a_{\varepsilon_{0}} u_{0}\right) \psi_{\text {orth }}=\hat{\psi}+\lambda \psi_{0}-2 a_{\varepsilon_{0}} \psi_{0}\left(\alpha \psi_{0}+u_{\text {orth }}\right) .
$$


This problem admits a solution if, and only if,

$$
\int_{\Omega} \hat{\psi} \psi_{0}+\lambda \int_{\Omega} \psi_{0}^{2}-2 \alpha \int_{\Omega} a_{\varepsilon_{0}} \psi_{0}^{3}-2 \int_{\Omega} a_{\varepsilon_{0}} \psi_{0}^{2} u_{\text {orth }}=0 .
$$

Moreover, if (5.12) holds, then (5.11) possesses a unique solution $\psi_{\text {orth }} \in X$. Thanks to Propositions 4.1 and 4.2 , we have

$$
\int_{\Omega} a_{\varepsilon_{0}} \psi_{0}^{3} \neq 0
$$

Therefore, there exists a unique value of $\alpha$ satisfying (5.12). This shows that (5.4) is an isomorphism.

Now, it follows from the implicit function theorem that there exists $\delta>0$ and a unique analytic mapping

$$
(u[\cdot], \psi[\cdot], \lambda[\cdot]):\left(\varepsilon_{0}-\delta, \varepsilon_{0}+\delta\right) \rightarrow U^{2} \times \mathbb{R}
$$

such that

$$
\left(u\left[\varepsilon_{0}\right], \psi\left[\varepsilon_{0}\right], \lambda\left[\varepsilon_{0}\right]\right)=\left(u_{0}, \psi_{0}, \lambda_{0}\right)
$$

and

$$
\mathcal{F}_{T}(u[\varepsilon], \psi[\varepsilon], \lambda[\varepsilon], \varepsilon)=0, \quad\left|\varepsilon-\varepsilon_{0}\right|<\delta .
$$

Moreover, these are the unique zeros of $\mathcal{F}_{T}$ in a neighborhood of $\left(u_{0}, \psi_{0}, \lambda_{0}, \varepsilon_{0}\right)$, say $\mathcal{Q}$, and hence $(u[\varepsilon], \lambda[\varepsilon], \varepsilon),\left|\varepsilon-\varepsilon_{0}\right|<\delta$, are the unique degenerate solutions of (1.3) in the projection of $\mathcal{Q}$ on $U \times \mathbb{R}^{2}$. Notice that if $\delta$ is sufficiently small, then

$$
u[\varepsilon] \gg 0, \quad \psi[\varepsilon] \gg 0
$$

and hence Proposition 4.1 shows that $(u[\varepsilon], \lambda[\varepsilon])$ is a regular zero of $\mathcal{F}(\cdot, \cdot, \varepsilon)$. Let $(u[\varepsilon](s), \lambda[\varepsilon](s))$ denote the arc length parametrization of the curve of positive solutions of $\mathcal{F}(\cdot, \cdot, \varepsilon)=0$ passing through by $(u[\varepsilon], \lambda[\varepsilon])$, normalized so that

$$
(u[\varepsilon](0), \lambda[\varepsilon](0))=(u[\varepsilon], \lambda[\varepsilon]) .
$$

Proposition 4.1 implies

$$
\frac{d \lambda[\varepsilon]}{d s}(0)=0, \quad \frac{d u[\varepsilon]}{d s}(0)=\psi[\varepsilon] .
$$

Moreover,

$$
\frac{d^{2} \lambda[\varepsilon]}{d s^{2}}(0)=\frac{\int_{\Omega} a_{\varepsilon} \psi[\varepsilon]^{3}}{\int_{\Omega} u[\varepsilon] \psi[\varepsilon]} .
$$

On the other hand, we have

$$
\frac{\int_{\Omega} a_{\varepsilon} \psi[\varepsilon]^{3}}{\int_{\Omega} u[\varepsilon] \psi[\varepsilon]}=\frac{\int_{\Omega} a_{\varepsilon_{0}} \psi_{0}^{3}}{\int_{\Omega} u_{0} \psi_{0}}+O\left(\left|\varepsilon-\varepsilon_{0}\right|\right), \quad \varepsilon \rightarrow \varepsilon_{0},
$$

and, due to Proposition 4.2,

$$
\frac{\int_{\Omega} a_{\varepsilon_{0}} \psi_{0}^{3}}{\int_{\Omega} u_{0} \psi_{0}}<0
$$

Thus, if $\delta$ is sufficiently small,

$$
\frac{d^{2} \lambda[\varepsilon]}{d s^{2}}(0)<0 \quad \text { for } \quad\left|\varepsilon-\varepsilon_{0}\right|<\delta .
$$


Therefore, by Proposition $4.2,(u[\varepsilon], \lambda[\varepsilon])$ is a subcritical quadratic turning point of $\mathcal{F}(\cdot, \cdot, \varepsilon)=0$ and, in addition,

$$
\sigma_{1}^{\Omega}\left[\mathcal{L}-\lambda[\varepsilon](s)+2 a_{\varepsilon} u[\varepsilon](s)\right]>0 \quad \text { if } \quad s<0,
$$

and

$$
\sigma_{1}^{\Omega}\left[\mathcal{L}-\lambda[\varepsilon](s)+2 a_{\varepsilon} u[\varepsilon](s)\right]<0 \quad \text { if } \quad s>0 .
$$

Notice that the half-curve corresponding with $s<0$ can be reparametrized in terms of $\lambda$ and that the corresponding parametrization, say $u_{\varepsilon}(\lambda)$, is increasing with $\lambda$, because the principal eigenvalue of the linearization is positive.

Now, we show that if $\varepsilon$ is sufficiently close to $\varepsilon_{0}$, then $(u[\varepsilon], \lambda[\varepsilon])$ is the first degenerate solution on the curve of positive solutions of $\mathcal{F}(\cdot, \cdot, \varepsilon)=0$ bifurcating from $u=0$. To prove this fact we need the following result, whose proof can be accomplished in the same way as the proof of the exchange stability principle [9], by making explicit the $\varepsilon$-dependence in all the steps of the proof to be sure that all the eigenvalue perturbations are uniform in $\varepsilon$. Notice that $\varepsilon$ is bounded away from $\varepsilon^{*}$ if $\delta$ is sufficiently small.

Lemma 5.3. There exists a neighborhood $\mathcal{B}$ of $(u, \lambda)=\left(0, \sigma_{1}^{\Omega}[\mathcal{L}]\right)$ in $U \times \mathbb{R}$ such that if $\varepsilon$ is sufficiently close to $\varepsilon_{0}$, then any positive solution of $(1.3)$ in $\mathcal{B}$ is linearly asymptotically stable.

Let $B_{\rho}\left(0, \sigma_{1}^{\Omega}[\mathcal{L}]\right)$ denote the open ball of radius $\rho$ centered at $\left(0, \sigma_{1}^{\Omega}[\mathcal{L}]\right) \in U \times \mathbb{R}$ and $B_{\rho}\left(u_{0}, \lambda_{0}\right)$ the ball of radius $\rho$ centered at $\left(u_{0}, \lambda_{0}\right) \in U \times \mathbb{R}$. Choose $\rho$ small enough so that

$$
\bar{B}_{\rho}\left(0, \sigma_{1}^{\Omega}[\mathcal{L}]\right) \subset \mathcal{B}, \quad \bar{B}_{\rho}\left(u_{0}, \lambda_{0}\right) \subset \hat{\mathcal{Q}},
$$

where $\hat{\mathcal{Q}}$ is the $(u, \lambda)$-projection of $\mathcal{Q}$. Let $\lambda_{1}>\sigma_{1}^{\Omega}[\mathcal{L}]$ be such that

$$
\left(u\left(\lambda_{1}\right), \lambda_{1}\right) \in \partial B_{\rho}\left(0, \sigma_{1}^{\Omega}[\mathcal{L}]\right), \quad(u(\lambda), \lambda) \in B_{\rho}\left(0, \sigma_{1}^{\Omega}[\mathcal{L}]\right),
$$

for all $\lambda<\lambda_{1}$. Let $\lambda_{2}>\lambda_{1}$ be such that

$$
\left(u\left(\lambda_{2}\right), \lambda_{2}\right) \in \partial B_{\rho}\left(u_{0}, \lambda_{0}\right), \quad(u(\lambda), \lambda) \notin \bar{B}_{\rho}\left(u_{0}, \lambda_{0}\right),
$$

for $\lambda<\lambda_{2}$. Then,

$$
\mathcal{K}_{0}:=\left\{(u(\lambda), \lambda): \lambda_{1} \leq \lambda \leq \lambda_{2}\right\}
$$

is a compact arc of analytic curve such that

$$
\sigma_{1}^{\Omega}\left[\mathcal{L}-\lambda+2 a_{\varepsilon_{0}} u(\lambda)\right] \geq C>0, \quad \forall \lambda \in\left[\lambda_{1}, \lambda_{2}\right],
$$

for some positive constant $C$. The implicit function theorem together with a compactness argument show that if $\varepsilon$ is sufficiently close to $\varepsilon_{0}$, then the arc of curve $\mathcal{K}_{0}$ perturbs into a family of arcs

$$
\mathcal{K}_{\varepsilon}:=\left\{(u[\varepsilon](\lambda), \lambda): \lambda_{1}[\varepsilon] \leq \lambda \leq \lambda_{2}[\varepsilon]\right\}, \quad \varepsilon \simeq \varepsilon_{0},
$$

such that

$$
\lambda_{1}\left[\varepsilon_{0}\right]=\lambda_{1}, \quad \lambda_{2}\left[\varepsilon_{0}\right]=\lambda_{2}, \quad u\left[\varepsilon_{0}\right](\lambda)=u(\lambda) .
$$

By the continuous dependence of the principal eigenvalue on the potential we find that

$$
\sigma_{1}^{\Omega}\left[\mathcal{L}-\lambda+2 a_{\varepsilon_{0}} u[\varepsilon](\lambda)\right] \geq \frac{C}{2}>0,
$$


for all $\lambda \in\left[\lambda_{1}[\varepsilon], \lambda_{2}[\varepsilon]\right]$ if $\varepsilon$ is sufficiently close to $\varepsilon_{0}$. Moreover,

$$
\left(u[\varepsilon]\left(\lambda_{1}[\varepsilon]\right), \lambda_{1}[\varepsilon]\right) \in \mathcal{B}, \quad\left(u[\varepsilon]\left(\lambda_{2}[\varepsilon]\right), \lambda_{2}[\varepsilon]\right) \in \hat{\mathcal{Q}} .
$$

In $\mathcal{B}$ all the positive solutions are linearly asymptotically stable and in $\hat{\mathcal{Q}}$ the only degenerate solutions are $(u[\varepsilon], \lambda[\varepsilon])$. Therefore, $(u[\varepsilon], \lambda[\varepsilon])$ must be the first degenerate solution along the curve of positive solutions bifurcating from zero.

To complete the proof it suffices to show (iv). Differentiating $\mathcal{F}(u[\varepsilon], \lambda[\varepsilon], \varepsilon)=0$ with respect to $\varepsilon$ yields

$$
\left(\mathcal{L}-\lambda[\varepsilon]+2 a_{\varepsilon} u[\varepsilon]\right) \frac{d u}{d \varepsilon}[\varepsilon]=\frac{d \lambda}{d \varepsilon}[\varepsilon] u[\varepsilon]+a^{-} u^{2}[\varepsilon] .
$$

This problem admits a solution if, and only if,

$$
\frac{d \lambda}{d \varepsilon}[\varepsilon] \int_{\Omega} u[\varepsilon] \psi[\varepsilon]+\int_{\Omega} a^{-} u^{2}[\varepsilon] \psi[\varepsilon]=0,
$$

and therefore,

$$
\frac{d \lambda}{d \varepsilon}[\varepsilon]=-\frac{\int_{\Omega} a^{-} u^{2}[\varepsilon] \psi[\varepsilon]}{\int_{\Omega} u[\varepsilon] \psi[\varepsilon]}<0
$$

This completes the proof.

As a consequence of the previous result we obtain the following.

Theorem 5.4. Let $\varepsilon_{*}$ be the infimum of the set of values $\varepsilon_{0}<\varepsilon^{*}$ such that the turning point $(u[\varepsilon], \lambda[\varepsilon])$ is well defined for each $\varepsilon \in\left[\varepsilon_{0}, \varepsilon^{*}\right)$. Then,

$$
\lim _{\varepsilon \downarrow \varepsilon_{*}}\|u[\varepsilon]\|_{\infty}=\infty .
$$

Proof. By the local analysis of Section $3 \varepsilon_{*}$ is well defined. Moreover, $\varepsilon_{*} \geq 0$. To show (5.14) we argue by contradiction assuming that along some decreasing sequence $\varepsilon_{n}$ converging to $\varepsilon_{*}$ the sequence $u\left[\varepsilon_{n}\right]$ is bounded in $C_{0}(\bar{\Omega})$. We will get a contradiction with the definition of $\varepsilon_{*}$.

Since (1.3) is equivalent to a fixed point equation for a compact operator, we can extract a subsequence of $u\left[\varepsilon_{n}\right]$, relabeled by $n$, converging to a positive solution, say $u\left[\varepsilon_{*}\right]$, of (1.3) for $\varepsilon=\varepsilon_{*}$. Since $\lambda\left[\varepsilon_{n}\right]$ is increasing and bounded above by $\sigma_{1}^{\Omega \backslash \Omega_{+}}[\mathcal{L}]$, the limit

$$
\lambda\left[\varepsilon_{*}\right]:=\lim _{n \rightarrow \infty} \lambda\left[\varepsilon_{n}\right]
$$

is well defined. Moreover, for each $n \geq 1$ we have

$$
\sigma_{1}^{\Omega}\left[\mathcal{L}-\lambda\left[\varepsilon_{n}\right]+2 a_{\varepsilon} u\left[\varepsilon_{n}\right]\right]=0
$$

and hence, passing to the limit as $n \rightarrow \infty$ gives

$$
\sigma_{1}^{\Omega}\left[\mathcal{L}-\lambda\left[\varepsilon_{*}\right]+2 a_{\varepsilon} u\left[\varepsilon_{*}\right]\right]=0 .
$$

Thus, $\left(u\left[\varepsilon_{*}\right], \lambda\left[\varepsilon_{*}\right]\right)$ satisfies the assumptions of Proposition 4.1. Moreover, due to (4.5),

$$
\lambda_{2}\left[\varepsilon_{*}\right]=\frac{\int_{\Omega} a_{\varepsilon_{*}} \psi^{3}\left[\varepsilon_{*}\right]}{\int_{\Omega} u\left[\varepsilon_{*}\right] \psi\left[\varepsilon_{*}\right]}=\frac{\int_{\Omega} a_{\varepsilon} \psi^{3}[\varepsilon]}{\int_{\Omega} u[\varepsilon] \psi[\varepsilon]}+O\left(\left|\varepsilon-\varepsilon_{*}\right|\right), \quad \varepsilon \downarrow \varepsilon_{*},
$$

and therefore, it follows from Proposition 4.2 that $\left(u\left[\varepsilon_{*}\right], \lambda\left[\varepsilon_{*}\right]\right)$ is a quadratic subcritical turning point. 
It suffices to show that $\left(u\left[\varepsilon_{*}\right], \lambda\left[\varepsilon_{*}\right]\right)$ is the first singular point on the curve of positive solutions bifurcating from $u=0$ for (1.3) with $\varepsilon=\varepsilon_{*}$. Indeed, if this is the case, then it follows from Theorem 5.1 that the structure of this curve will be the same for $\varepsilon<\varepsilon_{*}$ sufficiently close and this contradicts the definition of $\varepsilon_{*}$.

For each $n \geq 1$, let $\Gamma_{n}^{+}$denote the curve of positive solutions of (1.3) with $\varepsilon=\varepsilon_{n}$ joining $\left(0, \sigma_{1}^{\Omega}[\mathcal{L}]\right)$ with $\left(u\left[\varepsilon_{n}\right], \lambda\left[\varepsilon_{n}\right]\right)$. Let $\left(u_{n}(\lambda), \lambda\right), \sigma_{1}^{\Omega}[\mathcal{L}] \leq \lambda \leq \lambda\left[\varepsilon_{n}\right]$, denote its $\lambda$-parametrization. Consider the set $\Gamma_{*}^{+}$of $\omega$-limits of $\Gamma_{n}^{+}, n \geq 1$. This set is closed and connected and hence it provides us with a continuum of solutions of (1.3) for $\varepsilon=\varepsilon_{*}$ joining $\left(0, \sigma_{1}^{\Omega}[\mathcal{L}]\right)$ with $\left(u\left[\varepsilon_{*}\right], \lambda\left[\varepsilon_{*}\right]\right)$. Moreover, for each $n \geq 1, u_{n}(\lambda)$ is a strict positive supersolution of (1.3) with $\varepsilon=0$ and so

$$
u_{n}(\lambda) \gg \theta_{\lambda} .
$$

Therefore, $\Gamma_{*}^{+}$is constituted by positive solutions of (1.3) for $\varepsilon=\varepsilon_{*}$. Furthermore, since

$$
\sigma_{1}^{\Omega}\left[\mathcal{L}-\lambda+2 a_{\varepsilon_{n}} u_{n}(\lambda)\right]>0, \quad n \geq 1, \quad \lambda \in\left(\sigma_{1}^{\Omega}[\mathcal{L}], \lambda\left[\varepsilon_{n}\right]\right),
$$

the following holds

$$
\sigma_{1}^{\Omega}\left[\mathcal{L}-\lambda+2 a_{\varepsilon_{*}} u\right] \geq 0
$$

for each $(u, \lambda) \in \Gamma_{*}^{+}$. By the uniqueness obtained as an application of the theorem by Crandall and Rabinowitz it follows from the exchange stability principle that in a neighborhood of the bifurcation point $\left(0, \sigma_{1}^{\Omega}[\mathcal{L}]\right)$ the continuum $\Gamma_{*}^{+}$possesses the structure of an analytic curve, say $\left(u_{*}(\lambda), \lambda\right), \lambda \simeq \sigma_{1}^{\Omega}[\mathcal{L}]$, such that

$$
\sigma_{1}^{\Omega}\left[\mathcal{L}-\lambda+2 a_{\varepsilon_{*}} u_{*}(\lambda)\right]>0 .
$$

The implicit function theorem guarantees that we can continue this curve as soon as we have (5.16). We claim that $\left(u\left[\varepsilon_{*}\right], \lambda\left[\varepsilon_{*}\right]\right)$ is the first degenerate solution on $\Gamma_{*}^{+}$. On the contrary, assume that the first singular point along $\Gamma_{*}^{+}$is

$$
\left(u_{*}, \lambda_{*}\right) \neq\left(u\left[\varepsilon_{*}\right], \lambda\left[\varepsilon_{*}\right]\right) .
$$

Then, by Propositions 4.1 and $4.2,\left(u_{*}, \lambda_{*}\right)$ is a subcritical quadratic turning point of $\Gamma_{*}^{+}$where the asymptotically stable solutions become unstable, but still being regular solutions of the curve, and therefore, these unstable solutions must be in $\Gamma_{*}^{+}$. This contradicts (5.15) and shows that, in fact, $\Gamma_{*}^{+}$is an analytic curve filled in by asymptotically stable solutions joining the bifurcation point with $\left(u\left[\varepsilon_{*}\right], \lambda\left[\varepsilon_{*}\right]\right)$ which is its first degenerate solution. This completes the proof.

\section{Further RESUlts AND COMPARISON With OTHER RESUlts IN THE REFERENCES}

In our current situation, Theorem 1 of [5] reads as follows.

Theorem 6.1. Assume $\sigma_{1}^{\Omega}[\mathcal{L}]>0$. Then, problem (1.3) with $\lambda=0$ has a solution provided $N<4$.

This is so, because $N<4$ implies $2<\frac{N+2}{N-1}$ and therefore, due to Theorem 4 of [5], the positive solutions of (1.3) are uniformly bounded for $\lambda$ varying in compact subintervals of $[0, \infty)$. Using this fact together with the theory developed in this paper, we obtain the following sharper version of Theorem 6.1. 
Theorem 6.2. Under the conditions

$$
\sigma_{1}^{\Omega}[\mathcal{L}]>0, \quad N<4,
$$

the following assertions are true:

(i) If $\varepsilon<\varepsilon^{*}$, then there exists

$$
\lambda^{*} \in\left(\sigma_{1}^{\Omega}[\mathcal{L}], \sigma_{1}^{\Omega \backslash \bar{\Omega}_{+}}[\mathcal{L}]\right)
$$

such that (1.3) does not admit a positive solution if $\lambda>\lambda^{*}$ and it possesses at least one positive solution for each $\lambda \in\left[0, \lambda^{*}\right)$. Moreover, there exists

$$
\lambda[\varepsilon] \in\left(\sigma_{1}^{\Omega}[\mathcal{L}], \lambda^{*}\right]
$$

such that (1.3) possesses at least two solutions for each $\lambda \in\left(\sigma_{1}^{\Omega}[\mathcal{L}], \lambda[\varepsilon]\right)$ and, in addition, at least one of these solutions is linearly asymptotically stable.

(ii) If $\varepsilon \geq \varepsilon^{*}$, then (1.3) does not admit a positive solution for $\lambda>\sigma_{1}^{\Omega}[\mathcal{L}]$, and it possesses a positive solution for each $\lambda \in\left[0, \sigma_{1}^{\Omega}[\mathcal{L}]\right)$.

Proof. (i) Assume $\varepsilon<\varepsilon^{*}$. Theorem 3.4(ii) implies that the set $\Lambda$ of values $\lambda_{1}>$ $\sigma_{1}^{\Omega}[\mathcal{L}]$ such that $(1.3)$ has a positive solution for each $\lambda \in\left(\sigma_{1}^{\Omega}[\mathcal{L}], \lambda_{1}\right]$ is nonempty. Moreover, due to Theorem 2.3, it is bounded above by $\sigma_{1}^{\Omega \backslash \bar{\Omega}_{+}}[\mathcal{L}]$. Therefore, $\lambda^{*}:=$ $\sup \Lambda$ is well defined. We now show that (1.3) does not admit a positive solution if $\lambda>\lambda^{*}$. On the contrary, assume that (1.3) has a positive solution $u_{0}$ for some $\lambda_{0}>\lambda^{*}$. Then, $u_{0}$ is a supersolution of (1.3) for any $\lambda \leq \lambda_{0}$. Moreover, if $\delta$ is sufficiently small and $\varphi_{1}$ is the principal eigenfunction associated with $\sigma_{1}^{\Omega}[\mathcal{L}]$, then $\delta \varphi_{1}$ is a subsolution of $(1.3)$ provided $\lambda>\sigma_{1}^{\Omega}[\mathcal{L}]$. Thus, (1.3) possesses a positive solution for each $\lambda \in\left(\sigma_{1}^{\Omega}[\mathcal{L}], \lambda_{0}\right]$ and hence $\lambda_{0} \in \Lambda$. This contradicts the definition of $\lambda^{*}$. Therefore, (1.3) does not admit a positive solution if $\lambda>\lambda^{*}$. By construction, (1.3) possesses at least one positive solution if $\lambda \in\left(\sigma_{1}^{\Omega}[\mathcal{L}], \lambda^{*}\right)$.

Now, it will be shown that (1.3) possesses a positive solution for each $\lambda \in$ $\left[0, \sigma_{1}^{\Omega}[\mathcal{L}]\right]$. Let $\mathcal{C}^{+}$denote the global continuum of positive solutions bifurcating from $u=0$ at $\sigma_{1}^{\Omega}[\mathcal{L}]$. By the celebrated theorem of Rabinowitz [26] $\mathcal{C}^{+}$is unbounded in $C_{0}(\bar{\Omega}) \times \mathbb{R}$. By (6.1) and the results of [5] there exists $M=M(\varepsilon)$ such that $\|u\|_{\infty}<M$ for any positive solution $(u, \lambda)$ of $(1.3)$ with $\lambda \in\left[0, \lambda^{*}+1\right]$. Set

$$
\mathcal{O}:=\left\{(u, \lambda) \in C_{0}(\bar{\Omega}) \times \mathbb{R}:\|u\|_{\infty} \leq M, 0 \leq \lambda^{*}+1\right\} .
$$

This is a bounded and closed set whose interior contains the curve of positive solutions of $\mathcal{C}^{+}$bifurcating from $u=0$. Since $\mathcal{C}^{+}$is unbounded, there exists $\left(u_{1}, \lambda_{1}\right) \in \mathcal{C}^{+} \cap \partial \mathcal{O}$. We know that $\|u\|_{\infty}<M$ and that (1.3) admits a positive solution if $\lambda>\lambda^{*}$. Thus, $\lambda_{1}=0$. Moreover, since $\mathcal{C}^{+}$is connected, its projection on the $\lambda$ axis is an interval and since $\varepsilon<\varepsilon^{*}$ the bifurcation of $\mathcal{C}^{+}$is supercritical. Therefore, (1.3) possesses at least one solution for each $\lambda \in\left[0, \sigma_{1}^{\Omega}[\mathcal{L}]\right]$.

For values of $(u, \lambda)$ close to $\left(0, \sigma_{1}^{\Omega}[\mathcal{L}]\right), \mathcal{C}^{+}$has the structure of an analytic curve which can be parametrized by $\lambda,(u(\lambda), \lambda)$. Since $u(\lambda)$ is uniformly bounded, (4.27) is excluded and so this curve bends back at a quadratic turning point. In Section 5 denote it by $(u[\varepsilon], \lambda[\varepsilon])$. We have

$$
\left(u_{1}, 0\right),(u[\varepsilon], \lambda[\varepsilon]) \in \mathcal{C}^{+} .
$$

Let $\mathcal{C}_{j}^{+} \subset \mathcal{C}^{+}$denote any subcontinuum of $\mathcal{C}^{+}$joining $\left(u_{1}, 0\right)$ with $(u[\varepsilon], \lambda[\varepsilon])$. Since for any $\lambda \in\left(\sigma_{1}^{\Omega}[\mathcal{L}], \lambda[\varepsilon]\right)$ the solution $(u(\lambda), \lambda)$ does not degenerate, the following 
set is empty

$$
\mathcal{C}_{j}^{+} \cap\left\{(u(\lambda), \lambda), \quad \sigma_{1}^{\Omega}[\mathcal{L}]<\lambda<\lambda[\varepsilon]\right\} .
$$

Therefore, (1.3) possesses at least two positive solutions for each $\lambda \in\left(\sigma_{1}^{\Omega}[\mathcal{L}], \lambda[\varepsilon]\right)$. This completes the proof of part (i).

Part (ii) follows from Theorem 3.6 using the global bifurcation theorem as in the proof of part (i).

Note that thanks to (2.4) we find from Theorem 2.5 that $M(\varepsilon) \uparrow \infty$ as $\varepsilon \downarrow 0$, showing how deep are the results of Section 3 of [5]. In our setting Theorem 2 of [5] reads as follows:

Theorem 6.3. Assume

$$
\sigma_{1}^{\Omega}[\mathcal{L}]=0, \quad \varepsilon<\varepsilon^{*} .
$$

Then, there exists $\lambda^{*}>0$ such that for $\lambda \in\left(0, \lambda^{*}\right)$ problem (1.3) has a positive solution, but for $\lambda>\lambda^{*}$ it has no solution.

The argument in the begining of the proof of Theorem 6.2 provides us with the following sharper version.

Theorem 6.4. Assume $\varepsilon<\varepsilon^{*}$. Then, there exists $\lambda^{*}>0$ such that for $\lambda \in\left(0, \lambda^{*}\right)$ problem (1.3) has a positive solution, but for $\lambda>\lambda^{*}$ does not admit a positive solution.

Finally, Theorem 3 of 5 reads as follows.

Theorem 6.5. Under the conditions of Theorem 6.3 if in addition $N<4$, then (1.3) has a solution when $\lambda=0$.

With the same argument of the proof of our Theorem 6.2(i) we can prove the following sharper version.

Theorem 6.6. Assume

$$
\sigma_{1}^{\Omega}[\mathcal{L}]=0, \quad \varepsilon<\varepsilon^{*}, \quad N<4 .
$$

Then, there exists

$$
\lambda^{*} \in\left(0, \sigma_{1}^{\Omega \backslash \bar{\Omega}_{+}}[\mathcal{L}]\right)
$$

such that (1.3) does not admit a positive solution if $\lambda>\lambda^{*}$ and it possesses at least one positive solution if $\lambda \in\left[0, \lambda^{*}\right)$. Moreover, there exists

$$
\lambda[\varepsilon] \in\left(\sigma_{1}^{\Omega}[\mathcal{L}], \lambda^{*}\right]
$$

such that (1.3) possesses at least two solutions for each $\lambda \in\left(\sigma_{1}^{\Omega}[\mathcal{L}], \lambda[\varepsilon]\right)$; at least one of them is linearly asymptotically stable.

Now, we will compare our results with the theorems of [23. Our Theorem 2.4 is sharper than Theorem 1 of 23], which was the main theorem of 22. Our proof is considerably shorter and works out for general operators and general boundary conditions (cf. [14]). Moreover, we study, in addition, the stability properties of the solutions and show that $\theta_{\lambda}$ grows to infinity uniformly on compact subsets of $\Omega \backslash \bar{\Omega}_{+}$as $\lambda \uparrow \sigma_{1}^{\Omega \backslash \bar{\Omega}_{+}}[\mathcal{L}]$. In particular, any $L^{p}$-norm of $\theta_{\lambda}$ grows to infinity and not only the $L^{2}$-norm which is the result found in [22]. In our setting, the main Theorem of [23] reads as follows (cf. Theorem 2 in Section 1 and Theorem 3 in Section 4 of $[23]$ ). 
Theorem 6.7. If $0<\varepsilon<\varepsilon^{*}$, then

(1) There is a number $\lambda_{0} \in\left(\sigma_{1}^{\Omega}[\mathcal{L}], \sigma_{1}^{\Omega \backslash \bar{\Omega}_{+}}[\mathcal{L}]\right)$ such that for any $\lambda \in\left(\sigma_{1}^{\Omega}[\mathcal{L}], \lambda_{0}\right)$, there is a positive solution $u(\lambda)$ of Problem (1.3) which is the smallest solution of Problem (1.3). Moreover, $(u(\lambda), \lambda)$ forms a smooth increasing solution curve starting at $\left(0, \sigma_{1}^{\Omega}[\mathcal{L}]\right)$.

(2) For $N \leq 14$, the solution curve $(u(\lambda), \lambda)$ continues smoothly through $\left(u\left(\lambda_{0}\right), \lambda_{0}\right)$ and bends back in such a way that for each $\lambda$ in some deleted left neighborhood of $\lambda_{0}$, there are precisely two distinct positive solutions of Problem (1.3) near $\left(u\left(\lambda_{0}\right), \lambda_{0}\right)$.

(3) $\lambda_{0}$ is the greatest number for the solvability of Problem (1.3), i.e., for any $\lambda>\lambda_{0}$ there is no positive solution of (1.3). Moreover, for $\lambda=\lambda_{0}$, the positive solution $u\left(\lambda_{0}\right)$ of $(1.3)$ is unique.

(4) For $N \leq 6$, there exist two distinct positive solutions of Problem (1.3) for all $\lambda \in\left(\sigma_{1}^{\Omega}[\mathcal{L}], \lambda_{0}\right)$.

The fact that $u(\lambda)$ is the pointwise smallest positive solution of (1.3) was shown in Lemma 2 of [23], pp. 1092-1093, where we send for details. Condition $N \leq 14$ guarantees that $u(\lambda)$ is bounded in $C(\bar{\Omega})$ and therefore shows the existence of a first turning point on the curve bifurcating from $u=0$. This is a very interesting result as it is valid for any $\varepsilon>0$. Our analysis in Sections 2 and 5 shows that, independently on $N$, there exists $\varepsilon_{*} \in\left[0, \varepsilon^{*}\right)$ such that part (2) of Theorem 6.7 holds provided $\varepsilon \in\left(\varepsilon_{*}, \varepsilon^{*}\right)$ (cf. Theorem 5.4), and fails for $\varepsilon=\varepsilon_{*}$. By using our terminology, part (2) of Theorem 6.7 shows that if $N \leq 14$, then $\varepsilon_{*}=0$. So, both kind of results fit together very well to get sharper information on how the bifurcation diagrams of (1.3) vary as $\varepsilon$ decreases from $\varepsilon^{*}$.

Theorem 6.7(4) is an immediate consequence from part (2). It has the same proof as our Theorem 6.2. In [23], pp. 1118-1139, the author used variational methods instead of global bifurcation theory to prove it. Our proof is really striking and, in addition, gives the result for a larger range of dimensions; namely, $N \leq 14$.

\section{REFERENCES}

[1] S. Alama and M. Del Pino, Solutions of elliptic equations with indefinite nonlinearities via Morse theory and linking, Ann. Inst. Henri Poincaré 13 (1996), 95-115. MR 96m:35091

[2] H. Amann, Fixed point equations and nonlinear eigenvalue problems in ordered Banach spaces, SIAM Review 18 (1976), 620-709. MR 54:3519; 57; 7269

[3] H. Beresticky, I. Capuzzo-Dolcetta and L. Nirenberg, Problèmes elliptiques indéfinis et théorèmes de Liouville non linéaires, C. R. Acad. Sci. Paris Sér. I. Math. 317 (1993), 945-950. MR 94k:35094

[4] H. Beresticky, I. Capuzzo-Dolcetta and L. Nirenberg, Variational methods for indefinite superlinear homogeneous elliptic problems, Nonlinear Differential Equations Appl. 2 (1995), 553-572. MR 96i:35033

[5] H. Beresticky, I. Capuzzo-Dolcetta and L. Nirenberg, Superlinear indefinite elliptic problems and nonlinear Liouville theorems, Topol. Methods Nonlinear 4 (1994), 59-78. MR 96d:35041

[6] H. Beresticky, L. Nirenberg and S. R. S. Varadhan, The principal eigenvalue and maximum principle for second order elliptic operators in general domains, Comm. Pure and Appl. Maths. 47 (1994), 47-92. MR 95h:35053

[7] H. Brezis and L. Oswald, Remarks on sublinear elliptic equations, Nonl. Anal. TMA 10 (1986), 55-64. MR 87c:35057

[8] M. G. Crandall and P. H. Rabinowitz, Bifurcation from simple eigenvalues, J. Funct. Anal. 8 (1971), 321-340. MR 44:5836

[9] M. G. Crandall and P. H. Rabinowitz, Bifurcation, perturbation of simple eigenvalues and linearized stability, Arch. Rat. Mech. Anal. 52 (1973), 161-180. MR 49:5962 
[10] E. N. Dancer, Some remarks on classical problems and fine properties of Sobolev spaces, Diff. Int. Eqns. 9 (1996), 437-446. MR 97e:35057

[11] M. A. Del Pino, Positive solutions of a semilinear elliptic equation on a compact manifold, Nonl. Anal. TMA 22 (1994), 1423-1430. MR 95g:58256

[12] M. A. Del Pino and P. Felmer, Multiple solutions for a semilinear elliptic equation, Trans. Amer. Math. Soc. 347 (1995), 4839-4853. MR 96c:35062

[13] J. Esquinas and J. López-Gómez, Multiparameter bifurcation for some particular reaction diffusion systems, Proc. Roy. Soc. Edinburgh 112A (1989), 135-143. MR 90k:35022

[14] J. M. Fraile, P. Koch, J. López-Gómez and S. Merino, Elliptic eigenvalue problems and unbounded continua of positive solutions of a semilinear elliptic equation, J. Diff. Eqns. 127 (1996), 295-319. MR 97b:35036

[15] M. Golubitsky and D. G. Schaeffer, Singularities and Groups in Bifurcation Theory, Volume I, Springer, New York, 1985. MR 86e:58014

[16] H. B. Keller and Z. H. Yang, A direct method for computing higher order folds, SIAM J. Sci. Stat. Comput. 7 (1986), 351-361. MR 87h:58023

[17] R. Nagel et al., One-parameter semigroups of positive operators, Lecture Notes in Mathematics \#1184, Springer 1986. MR 88i:47022

[18] J. López-Gómez, On linear weighted boundary value problems, in "Partial Differential Equations; Models in Physics and Biology," (G. Lumer et al. eds.) Akademie Verlag, Berlin 1994, 188-203. MR 96b:35154

[19] J. López-Gómez, The maximum principle and the existence of principal eigenvalues for some linear weighted boundary value problems, J. Diff. Eqns. 127 (1996), 263-294. MR 97b:35037

[20] J. López-Gómez, On the uniqueness of positive solutions for a class of sublinear elliptic problems, Boll. Un. Mat. Italiana (1997), 697-711. MR 98i:35062

[21] J. López-Gómez and M. Molina-Meyer, The maximum principle for cooperative weakly coupled elliptic systems and some applications, Diff. Int. Eqns. 7 (1994), 383-398. MR 94k:35053

[22] T. Ouyang, On the positive solutions of semilinear equations $\Delta u+\lambda u-h u^{p}=0$ on the compact manifolds, Trans. Amer. Math. Soc. 331 (1992), 503-527. MR 92k:55012

[23] T. Ouyang, On the positive solutions of semilinear equations $\Delta u+\lambda u+h u^{p}=0$ on compact manifolds. Part II, Indiana Univ. J. 40 (1991), 1083-1141. MR 92m:35017

[24] M. Picone, Sui valori eccenzionali di un parametro da cui dipende un'equazione differenziale ordinaria del secondo ordine, Ann. Sc. Nor. Sup. Pisa 11 (1910), 1-141.

[25] M. H. Protter and H. F. Weinberger, Maximum Principles in Differential Equations, Englewood Cliffs, Prentice-Hall, N. J. 1967. MR 86f:35034 MR 36:2935

[26] P. H. Rabinowitz, Some global results for nonlinear eigenvalue problems, J. Funct. Anal. 7 (1971), 487-513. MR 46:745

Departamento de Matemática Aplicada, Universidad Complutense, 28040-Madrid, SPAIN

E-mail address: julian@sunma4.mat.ucm.es 